\title{
Controlling Electronic Transitions in Fullerene van der Waals Aggregates via Supra- Molecular Assembly
}

Saunak Das ${ }^{1,5}$, Felix Herrmann-Westendorf ${ }^{1,5}$, Felix H. Schacher ${ }^{2,3}$, Eric Täuscher ${ }^{4}$, Uwe Ritter $^{4}$, Benjamin Dietzek ${ }^{1,5}$, Martin Presselt ${ }^{1,5}{ }^{*}$

${ }^{1}$ Institute of Physical Chemistry, Friedrich Schiller University Jena, Helmholtzweg 4, 07743 Jena, Germany

${ }^{2}$ Institute of Organic Chemistry and Macromolecular Chemistry (IOMC), Friedrich Schiller University Jena, Humboldtstraße 10, Jena, 07743, Germany

${ }^{3}$ Jena Center for Soft Matter (JCSM), Friedrich Schiller University Jena, Philosophenweg 7 , Jena, 07743, Germany

${ }^{4}$ Institute for Chemistry and Biotechnology, Ilmenau University of Technology, D-98684 Ilmenau, Germany

5 Leibniz Institute of Photonic Technology (IPHT), Albert-Einstein-Str. 9, 07745 Jena, Germany

*Corresponding author: martin.presselt@uni-jena.de 


\section{Supporting Information}

\section{Contents}

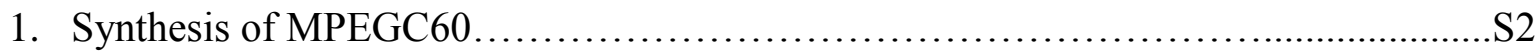

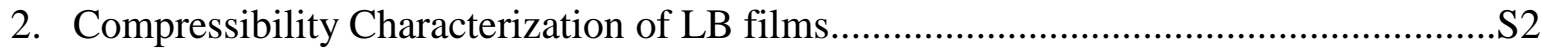

3. Dynamic Light Scattering Studies (DLS) ..........................................................S3

4. UV-vis spectra of solution dilution series..........................................................S4

5. Stepwise variation of MPEGC60 molecule for computational simplification...............S5

6. Electronic absorption spectra of $\mathrm{D}_{2 \mathrm{~h}}$ as a function of van der Waals distance (d)...... S9

7. Geometry of orbital overlap at the inter-fullerene junction.....................................S 9

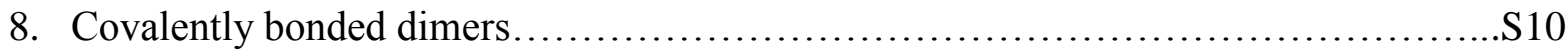

9. Total energies of optimized structures and intermonomeric distances........................S11

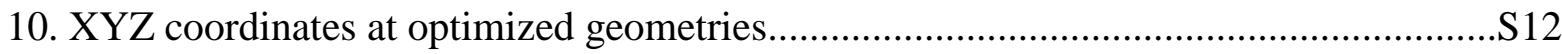

\section{Synthesis of PEGC60}

Synthesis of MPEGC60 (3' $H$-Cyclopropa[1,9][5,6]fullerene- $\mathrm{C}_{60}-I_{h}-3$ ',3'-dicarbonic acid-di\{2-[2-(2methoxyethoxy)ethoxy] ethylester was prepared according to the literature ${ }^{1}$ with some minor variations : A flame dried (Ar, Hg-bubbler) round bottom flask was charged with $\mathrm{C}_{60}(100 \mathrm{mg}, 139 \mu \mathrm{mol})$, bis- $\{2-[2-$ (2-methoxyethoxy)ethoxy]ethyl malonate, MPEG $(28.5 \mathrm{mg}, 72 \mathrm{mmol})$ and $90 \mathrm{~mL}$ dry toluene. Under stirring iodine ( $20 \mathrm{mg}, 78 \mu \mathrm{mol}$ ) was added at room temperature. After 5 minutes $20 \mu \mathrm{L} \mathrm{DBU}$ were added slowly. The obtained lilac solution was stirred overnight. TLC monitoring (toluene) confirmed that no $\mathrm{C}_{60}$ was present any more. The brown solution was filtered over silica, eluated with toluene and evaporated together with $2.5 \mathrm{~g}$ freshly added silica. The title compound was finally purified by flash-chromatography $\left(\mathrm{CH}_{2} \mathrm{Cl}_{2} /\right.$ methanol 40:1). The first fraction contained very little iodine, the second MPEGC60. After evaporation the product was freeze-dried (cyclohexane) and finally dried in vacuo (fluffy brown powder, $40-50 \%$ yields, the analytical data were in correspondence with the literature $)^{1-2}$. 

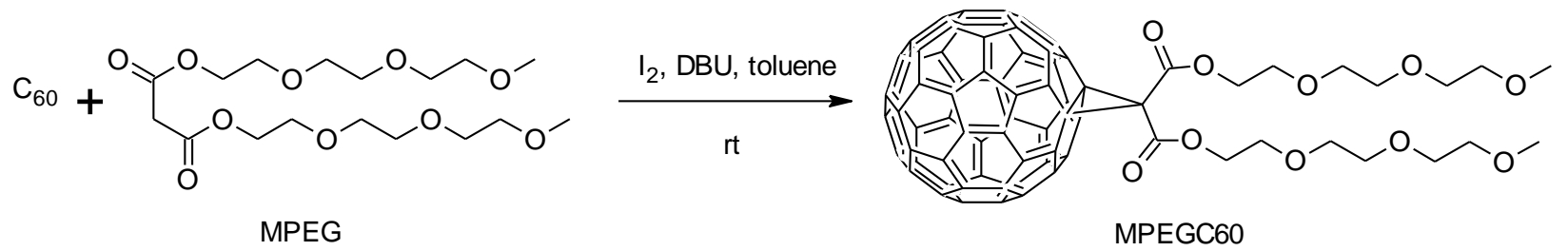

Figure S1: Synthesis of MPEGC60 from C60 and MPEG under specified reaction conditions

\section{Compressibility characterization of LB films}

An important parameter to get information about the rigidity of Langmuir films is the compressibility $C$, which can be calculated from a definite section of the $\Pi$-(A) isotherm taken at two different surface pressures preferably in the same phase given by the equation. ${ }^{3}$

$$
C=-\left(\frac{1}{A}\right) \frac{d A}{d \Pi}
$$

We calculated the compressibility of the Langmuir films choosing $\Pi_{1}$ and $\Pi_{2}$ as $5 \mathrm{mN} / \mathrm{m}$ and $30 \mathrm{mN} / \mathrm{m}$, respectively, with $\Pi_{1}$ to be the surface pressure at which our Langmuir-Blodgett films were deposited. We found that the compressibility of the pure MPEGC60 film is highest with $18.7 \mathrm{mN}^{-1}$ and minimum for pure stearic acid film with a value $12.5 \mathrm{mN}^{-1}$, as known for neutral fatty acid films. All compressibility values for the mixed films were low and lye within this range suggesting that MPEGC60 forms incompressible and rigid films in stearic acid matrix within $10-30 \mathrm{mN} / \mathrm{m}$ range of its $\Pi-\mathrm{A}$ isotherm.

\begin{tabular}{|c|c|}
\hline MPEGC60:stearic acid & Compressibility $\left(\mathbf{m N}^{-1}\right)$ \\
\hline $100: 0$ & 18.7 \\
$67: 33$ & 16.0 \\
$50: 50$ & 13.0 \\
$33: 67$ & 15.7 \\
$0: 100$ & 12.5 \\
\hline
\end{tabular}


Table S1: Calculated compressibility values for various MPEGC60:stearic acid Langmuir films

\section{Dynamic Light Scattering (DLS) Studies}

DLS studies were performed to analyze particle growth upon aggregation in MPEGC60: $\mathrm{CHCl}_{3}$ solutions. The particle size distributions shown in Figure S2 are governed by scattering effects from both solvation shells and the core C60-fullerenes with entangled MPEG chains. The mean particle distribution at the lowest concentration of $36 \mu \mathrm{M}(0.9 \%)$ is centered around $3.1 \mathrm{~nm}$ which slightly shifts to lower radii of around $2 \mathrm{~nm}$ on increasing the concentration to $48 \mu \mathrm{M}$ (1.3\%). The solvation shell of MPEGC60 in $\mathrm{CHCl}_{3}$ at such low concentration varies with the orientation of the flexible MPEG chains enveloping the C60 core. This variation in the solvent shell and might explain the difference between the mean radii of the two differently concentrated MPEGC60-solutions. Both radii approximately fit to the length to a single MPEGC60 molecule $(\sim 2.5 \mathrm{~nm})$ associated with a solvation shell. Therefore, both solutions are assumed to be dominated by MPEGC60 monomers.

At the $48 \mu \mathrm{M}$-solution, additionally we observe the appearance of the first onset of aggregation which was absent at $36 \mu \mathrm{M}$ concentration. The radial size distribution of these aggregates ranges from $4 \mathrm{~nm}$ over to $9 \mathrm{~nm}$ and is expected to be a signature from small van der Waals dimers $(\sim 5$ $\mathrm{nm})$ or trimers $(\sim 7.5 \mathrm{~nm})$ enveloped by the solvent shell. 


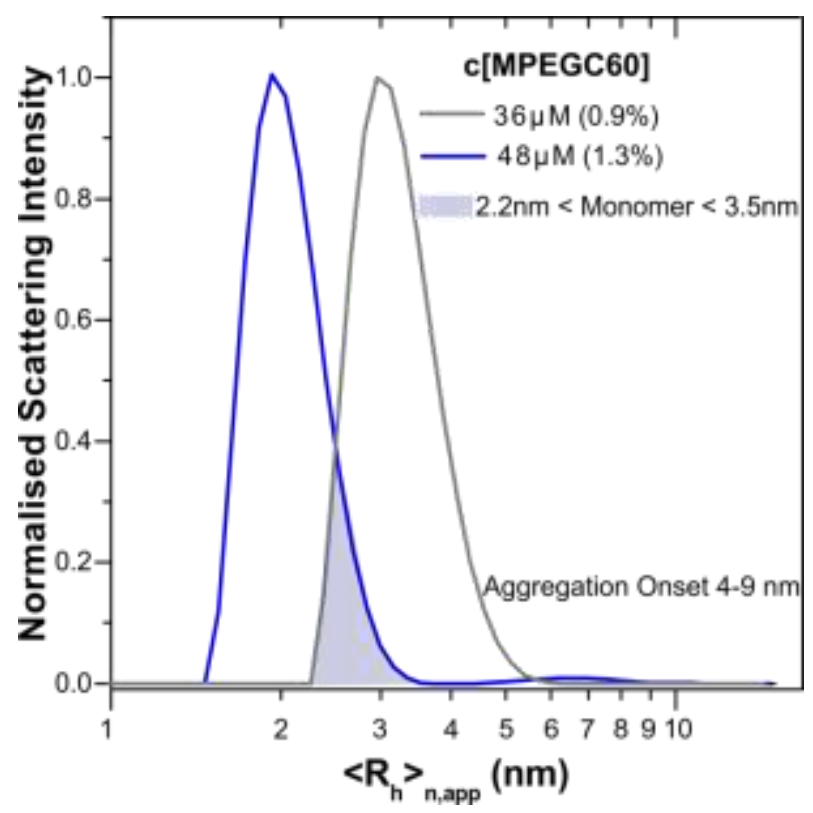

Figure S2: Dynamic light scattering data of MPEGC60: $\mathrm{CHCl}_{3}$ for the two lowest concentrations (36 $\mu \mathrm{M}$ and $48 \mu \mathrm{M})$

\section{4. $U V$-vis spectra of solution in a dilution series}

$\mathrm{UV}$-vis absorption spectra of MPEGC60/ $\mathrm{CHCl}_{3}$ solutions within the molar concentration range used to produce spin casted films did not show deviation from Lambert-Beers law as shown in Figure S3. Thus, aggregation in solution before spreading the solution on the Langmuir-Blodgett trough does not happen. In thin films the MPEGC60 molecules comes closer as the solvation shell disintegrates with $\mathrm{CHCl}_{3}$ evaporation and van der Waals aggregates can form. 


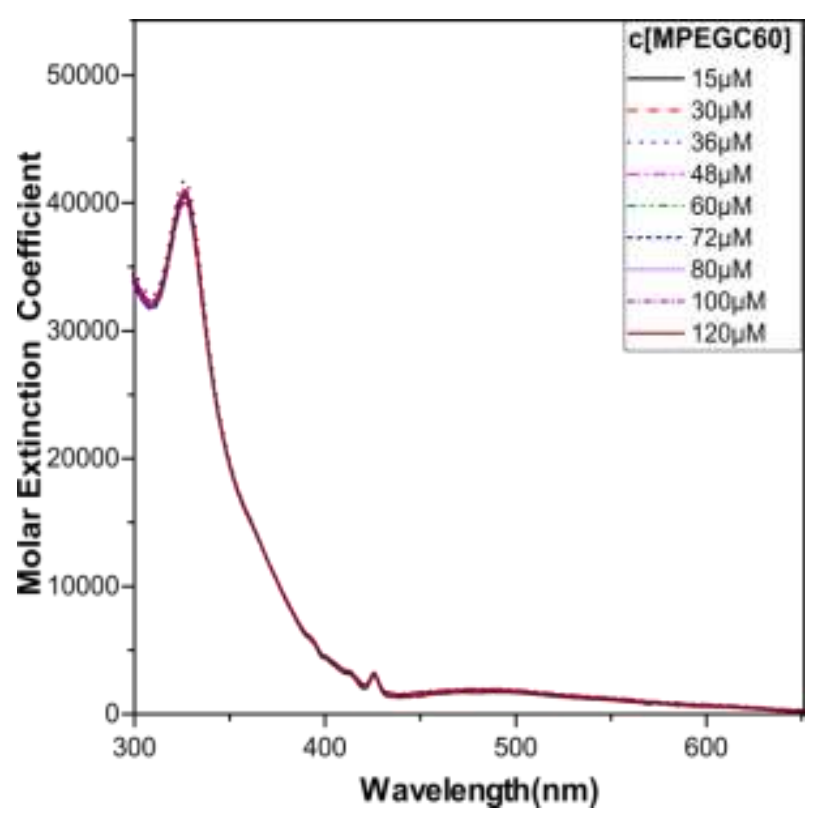

Figure S3: UV-vis absorption spectra of MPEGC60 in chloroform media

\section{Stepwise variation of parent MPEGC60 molecule for computational simplification}

Four test dimers of MPEGC60 (Figure S4) with approximate symmetries were chosen to test the feasibility of calculating their UV-vis absorption spectra by means of TD-DFT.

Since these calculations turned out to be computationally expensive, successive structural simplifications and changes of computational parameters were done as detailed in the following section (Figure S5). These simplifications finally allowed us to geometry-optimize a large number of dimers and higher aggregates with given symmetry and to calculate their UV-vis absorption spectra till the deep UV spectral range. At each step of modification we computed the theoretical absorption spectra with equal states for each irreducible representation. These modifications include

a. Replacing the MPEG chains with - $\mathrm{CN}$ and with - $\mathrm{CCH}$ substituents, which demonstrates that $\mathrm{CN}$ substituted $\mathrm{C60}$ is a better match for MPEGC60 than $-\mathrm{CCH}$ substituent. The electron withdrawing effect of $-\mathrm{CN}$ is closer to the ester derivative MPEG rather than the electron pushing effect of $-\mathrm{CCH}$ as demonstrated in the panel 'a' of Figure S5. So we computed $\mathrm{C}_{61}(\mathrm{CN})_{2}$-dimers instead of MPEGC60-dimers in the subsequent steps. 
b. The position of the bridgehead carbon was changed from between a pentagonal ring and hexagonal ring $[5,6]$, to between two hexagonal rings $[6,6]$. This modification enabled us to treat dimers of specific symmetries involving more than one mirror planes like $D_{2 h}$ or $D_{2 d}$ which is not possible in the other case. The results shown in panel ' $b$ ' of Figure S5 demonstrates that $[6,6]$ modification closely represents the absorption spectra of [5,6] modified system with a small deviation of the absorption spectra by $<10 \mathrm{~nm}$.

c. The third method modification was the use of symmetry which enabled us to compute a huge number of electronic transitions for several dimers at inexpensive computational cost. The effect of using symmetry shown in panel 'c' of Figure S5 finitely increases the resolution of the allowed absorption peak and diminishes the absorption in the symmetry forbidden vis-region. Hence we switched 'symmetry on' for subsequent computations.

d. Finally, the UV-vis absorption spectra obtained by using the two basis sets TZV and TZVP were compared in panel'd' of Figure S5. Both these basis sets could be excellent choices for simulation of absorption spectra. TZV basis was employed by Shubina ${ }^{4}$ and seemed a legitimate choice for treatment of van der Waals-dimers. Hence we performed our computations using TZV basis set. 

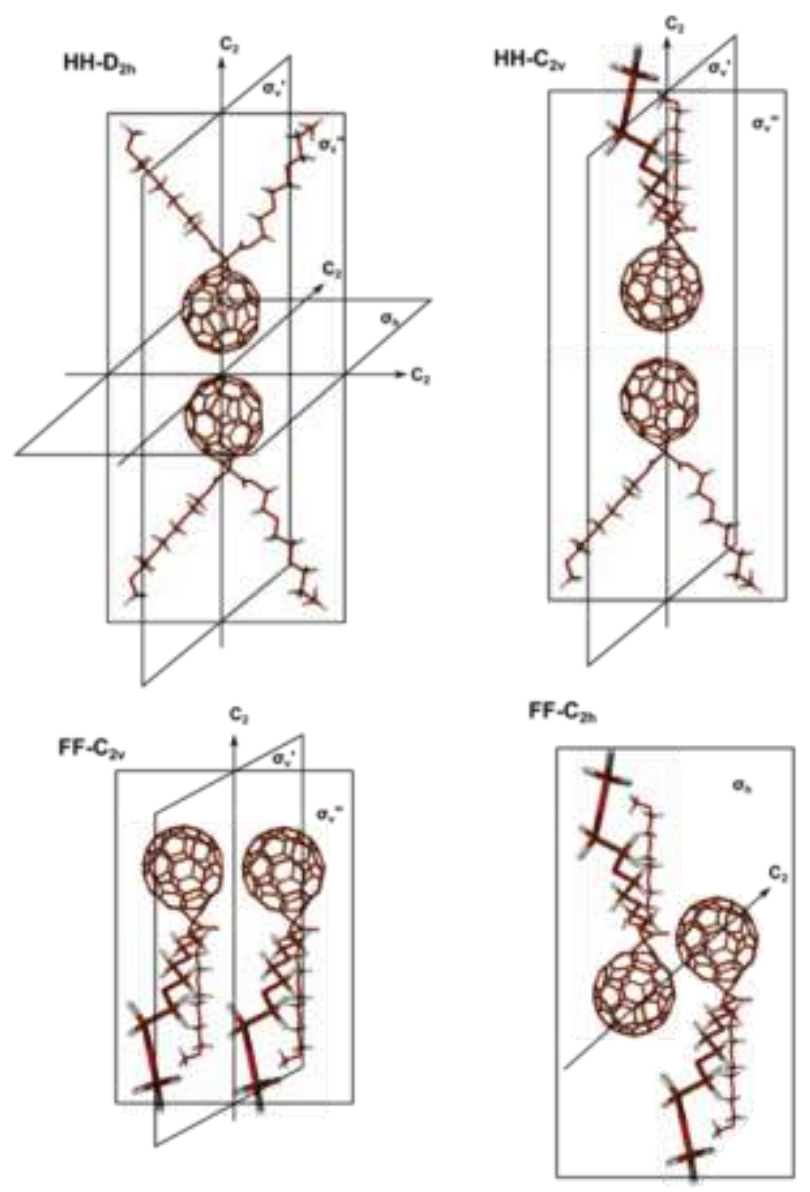

Figure S4: Four Test dimers for TDDFT simulation of absorption spectra 


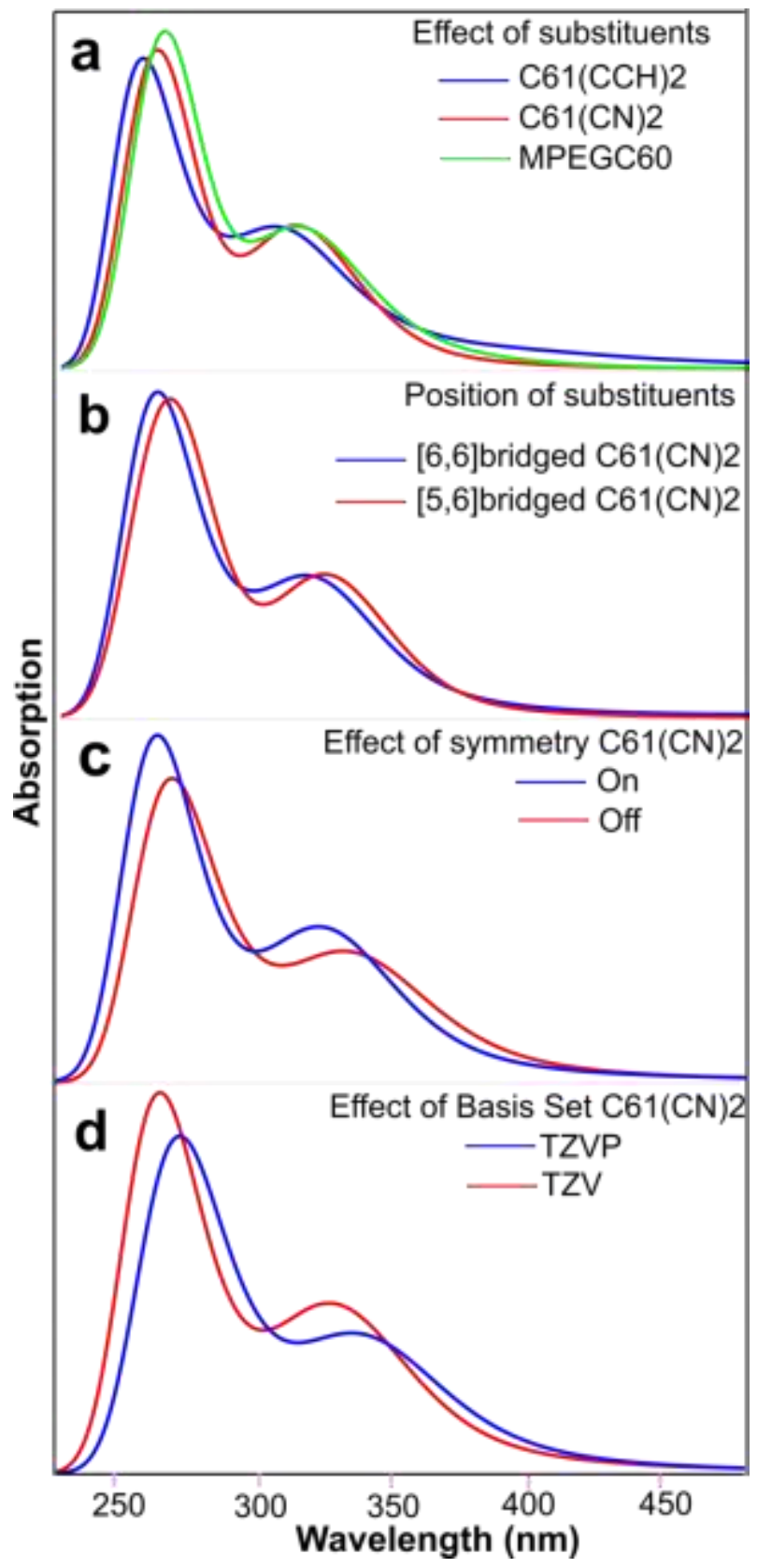

Figure S5: Stepwise molecular simplification and variation of computational parameters as detailed in preceding section: a) Effect of substituents, b) Position of substituents, c) Effect of symmetry, d) Effect of Basis Sets 


\section{Electronic absorption spectra of $1 D_{2 h}$ as a function of $v d W$ distance (d)}

The electronic absorption spectra of dimer $\mathrm{D}_{2 \mathrm{~h}}$ (Figure S6) is a classic case of the validation of Laporte's selection rule. As the van der Waals distance between the two $\mathrm{C}_{61}(\mathrm{CN})_{2}$ progressively diminishes the orbital overlap to generate collective states would be possible. But since the symmetry element ' $i$ ' is not withdrawn during distance variation, the centrosymmetric nature of the molecule is retained and forbidden visible absorption stay very weak. This molecular phenomenon is strikingly different from a non-centrosymmetric dimer $\mathrm{D}_{2 \mathrm{~d}}$ as detailed in the main text.

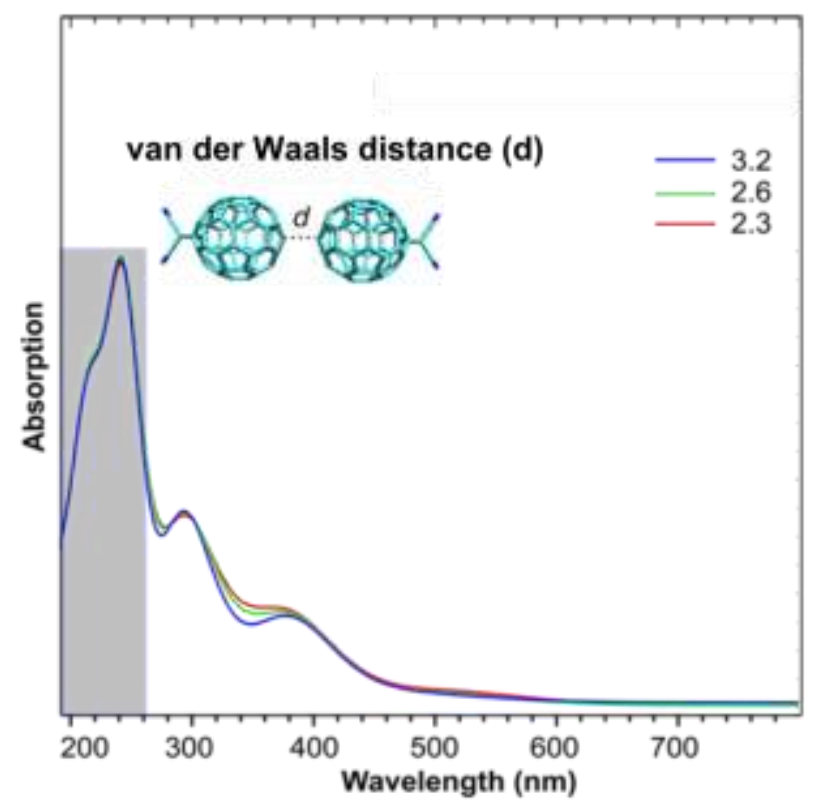

Figure S6: Electronic absorption spectra of $1 \mathrm{D}_{2 \mathrm{~h}}$ as a function of van der Waals distance (d)

\section{Geometry of orbital overlap at the inter-fullerene junction $\left(D_{2 h}, D_{2 d}\right)$}

Figure S7 distinguishes the geometry of overlap between the two bonds at the interfullerene junction in $D_{2 h}$ and $D_{2 h}$ dimers. Due to diagonal overlap the dimer $D_{2 d}$ is unable to form a covalently bonded dimer which can result in dimers with very small van der Waals distance and a huge visible absorption 


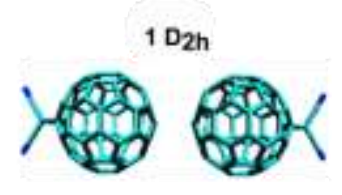

parallel overlap
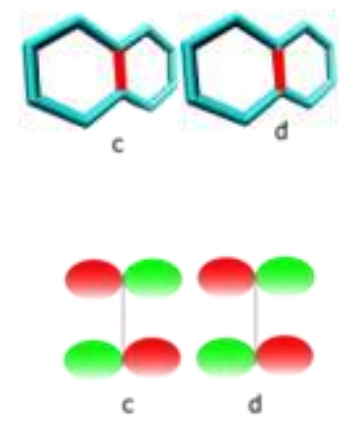

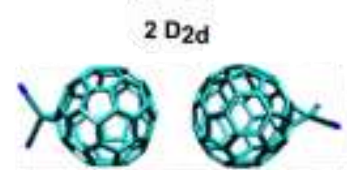

perpendicular overlap
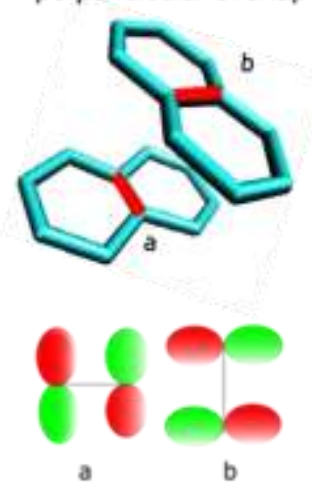

Figure S7: Geometry of orbital overlap at the interfullerene junction $\left(D_{2 h}, D_{2 d}\right)$.

\section{Covalently bonded dimers $\left(3 C_{2 v}, 1 D_{2 h}\right)$}

At van der Waals distances less than $2.6 \AA$ the 'p orbitals' at the junction overlaps in a parallel fashion for dimers like $D_{2 h}$ or $3 C_{2 v}$ shown in Figure S7. This results in formation of a [2+2] cycloaddition product ${ }^{5}$ which is commonly observed by photochemical ${ }^{6}$, electron-induced ${ }^{7}$, thermal and high-pressure or plasma based methods ${ }^{8}$ but is not synthetically straight forward. The spectral descriptions of these dimers are out of our current focus since they are not observable in Langmuir-Blodgett or Spin Coated thin solid films. 

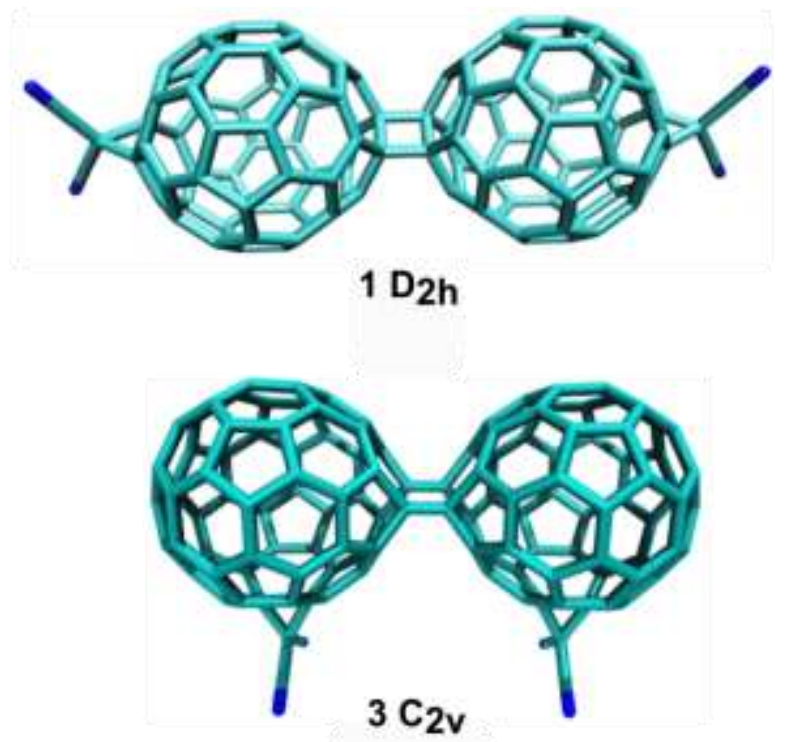

Figure S8: Covalently bonded dimers $\mathrm{D}_{2 \mathrm{~h}}$ and $3 \mathrm{C} 2 \mathrm{v}$ formed after geometry optimizing the molecule at a starting vdW distance $<3 \AA$

\section{Computed energies and inter-monomeric distances}

\begin{tabular}{|c|c|c|}
\hline Structure & $\begin{array}{c}\text { Relative Energy } \\
(\mathbf{k c a l} / \mathbf{m o l})\end{array}$ & $\begin{array}{c}\text { Intermolecular } \\
\text { Distance( } \mathbf{\AA})\end{array}$ \\
\hline $\mathrm{C}_{61}(\mathrm{CN})_{2}$ & 0 & - \\
\hline $1 \mathrm{D}_{2 \mathrm{~h}}$ & 2.714 & 3.2 \\
\hline $2 \mathrm{D}_{2 \mathrm{~d}}$ & 0.184 & 3.2 \\
\hline $3 \mathrm{C}_{2 \mathrm{v}}$ & -178.572 & 2.6 \\
\hline $4 \mathrm{C}_{2 \mathrm{~h}}$ & 4.301 & 3.2 \\
\hline $5 \mathrm{C}_{2 \mathrm{v}}$ & 0.989 & 3.2 \\
\hline $6 \mathrm{C}_{2 \mathrm{v}}$ & 1.081 & 3.2 \\
\hline $7 \mathrm{C}_{2 \mathrm{v}}$ & 2.254 & 3.2 \\
\hline $8 \mathrm{C}_{\mathrm{s}}$ & 2.53 & 2.6 \\
\hline $1 \mathrm{D}_{2 \mathrm{~h}}(\mathrm{~d}=2.6 \AA)$ & -161.828 & \\
\hline
\end{tabular}




\begin{tabular}{|c|c|c|}
\hline $1 \mathrm{D}_{2 \mathrm{~h}}(\mathrm{~d}=2.3 \AA)$ & -130.41 & 2.3 \\
\hline $1 \mathrm{D}_{2 \mathrm{~h}}(\mathrm{~d}=2 \AA)$ & -87.975 & 2 \\
\hline $2 \mathrm{D}_{2 \mathrm{~d}}(\mathrm{~d}=2.6 \AA)$ & -171.235 & 2.6 \\
\hline $2 \mathrm{D}_{2 \mathrm{~d}}(\mathrm{~d}=2.3 \AA)$ & -172.178 & 2.3 \\
\hline
\end{tabular}

Table S2: Total energies and inter-monomeric distances of the optimised molecules computed by TDDFT

10. Optimized XYZ coordinates from TDDFT output files

a. MPEGC60

117

C $\quad 4.1820850 \quad 4.3017515 \quad 1.5198804$

C $\quad 4.0037651 \quad 2.9364924 \quad 1.2870953$

C $\quad 2.8993559 \quad 2.2331492 \quad 1.9196701$

C $\quad 2.0162084 \quad 2.9052734 \quad 2.7589282$

C $\quad 2.2181603 \quad 4.3216653 \quad 3.0229523$

C $\quad 3.2733745 \quad 5.0074836 \quad 2.4095975$

C $\quad 2.3830789 \quad 1.2569047 \quad 0.9955760$

C $\quad \begin{array}{llll}\text { C } & 0219026 & 0.9197416 & 0.9429909\end{array}$

C $\quad 0.0636436 \quad 1.6781579 \quad 1.7691774$

C $\quad 0.5901683 \quad 2.6373993 \quad 2.6711771$

$\begin{array}{lllll}\text { C } & 0.5007004 & 0.3983832 & -0.3189137\end{array}$

C $\quad 1.1206505 \quad 0.8418780 \quad-1.4951973$

C $\quad 2.5119011 \quad 1.2197878 \quad-1.4455394$

C $\quad 3.1682158 \quad 1.3488557 \quad-0.2231773$

$\begin{array}{llll}\text { C } & 0.4837898 & 1.6215889 & -2.5634919\end{array}$ 

C $\quad 1.5620521 \quad 2.3707355 \quad-3.2273389$
C $\quad 2.8093499 \quad 2.1075106 \quad-2.5399771$
$\begin{array}{llll}\text { C } & -0.7968893 & 2.2250334 & -2.4628031\end{array}$
C $\quad-0.9806753 \quad 3.5729470 \quad-3.0260721$
C $\quad 0.0531425 \quad 4.2548626 \quad-3.6698991$
$\begin{array}{llll}\text { C } & 1.3570509 & 3.6382259 & -3.7729947\end{array}$
$\begin{array}{llll}\text { C } & 0.2439900 & 5.6830214 & -3.4594869\end{array}$
$\begin{array}{llll}\text { C } & -0.6148406 & 6.3918553 & -2.6137418\end{array}$
C $\quad-1.7041933 \quad 5.6956999 \quad-1.9574482$
$\begin{array}{llll}\text { C } & -1.8739891 & 4.3240761 & -2.1684618\end{array}$
C $\quad 1.6731046 \quad 5.9417916 \quad-3.4241329$
$\begin{array}{llll}\text { C } & 2.1915020 & 6.8876747 & -2.5308485\end{array}$
$\begin{array}{llll}\text { C } & 1.2990317 & 7.6085603 & -1.6380509\end{array}$
$\begin{array}{llll}\text { C } & -0.0781199 & 7.3676520 & -1.6801298\end{array}$
C $\quad-0.8383228 \quad 7.2701658 \quad-0.4436082$
$\begin{array}{llll}\text { C } & -0.1898599 & 7.4149917 & 0.7889574\end{array}$
$\begin{array}{llll}\text { C } & 1.2415567 & 7.6631157 & 0.8324853\end{array}$
$\begin{array}{llll}\text { C } & 1.9713000 & 7.7616512 & -0.3578753\end{array}$
C $3.2812917 \quad 7.1412336-0.4613466$
C $3.4192600 \quad 6.6047435 \quad-1.8054723$
C $\quad-1.8487893 \quad 6.2403298 \quad-0.6169792$
$\begin{array}{llll}\text { C } & 2.3635996 & 4.6802088 & -3.6275132\end{array}$
$\begin{array}{llll}\text { C } & 3.5545822 & 4.4185282 & -2.9439544\end{array}$
$\begin{array}{lllll}\text { C } & 4.0897741 & 5.3947497 & -2.0100582\end{array}$
C $\quad-2.1772526 \quad 5.3955486 \quad 0.4509575$
$\begin{array}{llll}\text { C } & -2.3845501 & 3.9775599 & 0.2170963\end{array}$
$\begin{array}{llll}\text { C } & -2.2303099 & 3.4647880 & -1.0695785\end{array}$
C $-1.6569996 \quad 2.1573571 \quad-1.2722956$
$\begin{array}{llll}\text { C } & -1.8089135 & 3.2408744 & 1.3281796\end{array}$
C $\quad-1.2252472 \quad 1.9834253 \quad 1.1223522$
$\begin{array}{llll}\text { C } & -1.4629985 & 1.3266410 & -0.1597318\end{array}$ 

C $\quad 3.7848069 \quad 3.0974775 \quad-2.3921545$
C $\quad 4.4669654 \quad 3.2504851 \quad-1.1165505$
$\begin{array}{llll}\text { C } & 4.1595999 & 2.3954110 & -0.0505485\end{array}$
$\begin{array}{llll}\text { C } & -0.9227715 & -0.0600967 & -0.3078277\end{array}$
C $\quad-0.0845264 \quad 3.9001519 \quad 2.9268505$
$\begin{array}{llll}\text { C } & -1.2608587 & 4.2030942 & 2.2496760\end{array}$
C $\quad 0.9132314 \quad 4.9393604 \quad 3.1265657$
C $\quad 4.5133697 \quad 5.1884058 \quad 0.4169606$
C $\quad 4.6493303 \quad 4.6723218 \quad-0.8778195$
$\begin{array}{llll}\text { C } & 0.7026683 & 6.2248457 & 2.6136361\end{array}$
$\begin{array}{llll}\text { C } & -0.5226886 & 6.5295871 & 1.8927499\end{array}$


C $\quad \begin{array}{llll}\text { C } & 1.7919760 & 6.9321406 & 1.9630061\end{array}$
$\begin{array}{llll}\text { C } & 3.0536321 & 6.3352981 & 1.8631668\end{array}$
C $\quad 3.8149148 \quad 6.4451496 \quad 0.6289362$
C $\quad-1.2875406 \quad-0.9785051 \quad 0.8907548$
$\begin{array}{llll}\text { O } & -0.3932643 & -1.9850813 & 0.9785477\end{array}$
$\begin{array}{llll}\text { C } & -0.6437517 & -2.9544407 & 2.0317605\end{array}$
C $\quad 0.4780076 \quad-3.9763235 \quad 1.9533766$
$\begin{array}{llll}\text { O } & 0.2168548 & -4.9398906 & 2.9618275\end{array}$
C $\quad \begin{array}{llll}1.2108571 & -5.9592233 & 3.0169101\end{array}$
C $\quad 0.8179912 \quad-6.9224908 \quad 4.1267206$
$\begin{array}{llll}\text { O } & 1.8097442 & -7.9384503 & 4.1816686\end{array}$
$\begin{array}{llll}\text { C } & 1.5464659 & -8.9087341 & 5.1895468\end{array}$
C $\quad 2.6868947 \quad-9.9269210 \quad 5.1607074$
$\begin{array}{llll}\text { O } & 2.5267061 & -10.9404199 & 6.1473877\end{array}$
C $\quad 1.6751398-12.0047683 \quad 5.7422056$
C $-1.3651096-0.8345746-1.5738676$
$\begin{array}{llll}\text { O } & -2.7102670 & -0.7721497 & -1.6893567\end{array}$
$\begin{array}{llll}\text { C } & -3.3017859 & -1.4833467 & -2.8106774\end{array}$
$\begin{array}{llll}\text { C } & -3.6834685 & -2.8970144 & -2.3900714\end{array}$ 


$$
\begin{aligned}
& \begin{array}{lllll}
\text { O } & -4.3666079 & -3.4781228 & -3.4900973
\end{array} \\
& \text { C } \quad-4.7578713 \quad-4.8251276 \quad-3.2410594 \\
& \text { C } \quad-5.4785930 \quad-5.3305954 \quad-4.4812575 \\
& \text { O } \\
& \text { C } \quad-6.5404665 \quad-7.2630937 \quad-5.3403670 \\
& \text { C } \quad-6.8959278 \quad-8.7000668-4.9577562 \\
& \text { O } \\
& \begin{array}{llll}
\text { C } & -6.7598029 & -9.9306077 & -6.9937078
\end{array} \\
& \begin{array}{llll}
\text { O } & -2.2554347 & -0.8392504 & 1.6017646
\end{array} \\
& \text { O } \\
& \text { H } \quad-0.6569587 \quad-2.4432137 \quad 3.0038839 \\
& \text { H } \quad-1.6248933 \quad-3.4232727 \quad 1.8729477 \\
& \text { H } \quad 0.4995740 \quad-4.4452372 \quad 0.9507879 \\
& \text { H } \quad 1.4563639 \quad-3.4846263 \quad 2.1170348 \\
& \text { H } \quad-4.1947585 \quad-0.9059813 \quad-3.0781462 \\
& \text { H } \quad-2.5965561-1.5017421 \quad-3.6505016 \\
& \text { H } \quad-2.7750158 \quad-3.4776595 \quad-2.1387650
\end{aligned}
$$

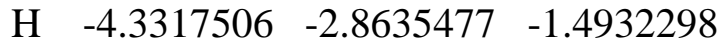

$$
\begin{aligned}
& \text { H } \quad-3.8734712 \quad-5.4575807 \quad-3.0346804 \\
& \text { H } \quad-5.4293265 \quad-4.8823876 \quad-2.3632238 \\
& \text { Н } 1.2731129 \quad-6.4982809 \quad 2.0524906 \\
& \text { H } \quad 2.2063619 \quad-5.5245372 \quad 3.2281978 \\
& \text { H } \quad 0.7528160 \quad-6.3809969 \quad 5.0901242 \\
& \text { H } \quad-0.1792748 \quad-7.3548951 \quad 3.9145281 \\
& \text { H } \quad-6.3687628 \quad-4.7038727 \quad-4.6828835 \\
& \text { H } \quad-4.8089182 \quad-5.2582237 \quad-5.3601594 \\
& \text { H } \quad-7.4655510 \quad-6.6994677 \quad-5.5696717 \\
& \text { H } \quad-5.9009611 \quad-7.2418462 \quad-6.2445443 \\
& \text { H } \quad-5.9764021 \quad-9.2507150 \quad-4.6824624 \\
& \text { H } \quad-7.5591804 \quad-8.6882330 \quad-4.0807250 \\
& \begin{array}{llll}
\text { H } & 1.4961489 & -8.4344677 & 6.1884486
\end{array}
\end{aligned}
$$




$$
\begin{array}{cccc}
\mathrm{H} & 0.5720239 & -9.4011710 & 5.0009775 \\
\mathrm{H} & 2.7682742 & -10.3663549 & 4.1486953 \\
\mathrm{H} & 3.6342385 & -9.4156486 & 5.3858943 \\
\mathrm{H} & -7.4110959 & -10.4674482 & -7.6946173 \\
\mathrm{H} & -6.1967732 & -9.1625174 & -7.5538654 \\
\mathrm{H} & -6.0382221 & -10.6445960 & -6.5539519 \\
\mathrm{H} & 1.6857548 & -12.7448839 & 6.5521068 \\
\mathrm{H} & 0.6326646 & -11.6774322 & 5.5784066 \\
\mathrm{H} & 2.0442383 & -12.4806519 & 4.8144808
\end{array}
$$

\section{b. PCBM}

85
C $\quad 8.5336963 \quad 10.5825134 \quad 8.0431487$
C $\quad 8.1307221 \quad 9.2736064 \quad 7.7667543$
$\begin{array}{llll}\text { C } & 6.7548350 & 8.8736381 & 8.0116537\end{array}$
$\begin{array}{llll}\text { C } & 5.8322067 & 9.7809147 & 8.5227082\end{array}$
C $\quad 6.2565626 \quad 11.1367832 \quad 8.8331473$
C $\quad 7.5779340 \quad 11.5321274 \quad 8.5909479$
C $\quad 6.3453354 \quad 7.9655638 \quad 6.9695084$
$\begin{array}{llll}\text { C } & 5.0334351 & 7.9296438 & 6.4809870\end{array}$
C $4.0694373 \quad 8.9328266 \quad 6.9559745$
C $\quad 4.4851385 \quad 9.8198841 \quad 7.9784159$
C $\quad 4.8088501 \quad 7.4412779 \quad 5.1159257$
C $\quad 5.8628719 \quad 7.6479045 \quad 4.2038703$
C $\quad 7.2194221 \quad 7.7181728 \quad 4.7019907$
C $\quad 7.4729844 \quad 7.7942810 \quad 6.0692178$
$\begin{array}{llll}\text { C } & 5.8037224 & 8.4579782 & 2.9843361\end{array}$
$\begin{array}{llll}\text { C } & 7.1767110 \quad 8.9058077 & 2.7058808\end{array}$
C $\quad 8.0447994 \quad 8.4334970 \quad 3.7623705$
$\begin{array}{llll}\text { C } & 4.7310818 & 9.3306463 & 2.6643077\end{array}$ 

$\begin{array}{llll}\text { C } & 5.0427463 & 10.6383615 & 2.0700386\end{array}$
C $\quad 6.3529527 \quad 11.0316759 \quad 1.7934675$
C $\quad 7.4468983 \quad 10.1431174 \quad 2.1202868$
$\begin{array}{llll}\text { C } & 6.7860451 & 12.3970642 & 2.0528920\end{array}$
C $\quad \begin{array}{llll}5.8880101 & 13.3336153 & 2.5745079\end{array}$
C $\quad 4.5191851 \quad 12.9374242 \quad 2.8451559$
C $\quad 4.1177341 \quad 11.6214066 \quad 2.5929522$
$\begin{array}{llll}\text { C } & 8.1501409 & 12.3450548 & 2.5490220\end{array}$
C $\quad 8.5610189 \quad 13.2219020 \quad 3.5606277$
C $\quad 7.6207323 \quad 14.1816301 \quad 4.1152511$
C $\quad 6.3102493 \quad 14.2387797 \quad 3.6300705$
C $\quad 5.1983334 \quad 14.3992388 \quad 4.5545440$
C $\quad 5.4409581 \quad 14.4941969 \quad 5.9299054$
C $\quad 6.8030003 \quad 14.4338067 \quad 6.4341443$
C $\quad 7.8723533 \quad 14.2835690 \quad 5.5435534$
C $\quad 8.9717703 \quad 13.3916419 \quad 5.8706062$
C $\quad 9.4001073 \quad 12.7378748 \quad 4.6447574$
C $\quad 4.0880546 \quad 13.5988075 \quad 4.0653314$
C $\quad 8.5638636 \quad 10.9526502 \quad 2.5827362$
C $\quad 9.3858526 \quad 10.4930741 \quad 3.6162912$
C $\quad 9.8076610 \quad 11.4001903 \quad 4.6710188$
C $\quad 3.2588967 \quad 12.9244646 \quad 4.9706060$
C $\quad 2.8213884 \quad 11.5697721 \quad 4.6847674$
C $\quad 3.2531882 \quad 10.9384353 \quad 3.5208059$
C $\quad 3.5545882 \quad 9.5235382 \quad 3.5153747$
C $\quad 2.8361450 \quad 10.8125046 \quad 5.9253529$
C $\quad 3.1599484 \quad 9.4497992 \quad 5.9229109$
C $\quad 3.2075216 \quad 8.7457743 \quad 4.6364883$
C $\quad 9.1231078 \quad 9.1996500 \quad 4.2159095$
C $\quad 9.3856316 \quad 9.2988039 \quad 5.6419998$
$\begin{array}{llll}\text { C } & 8.5709798 & 8.6128414 & 6.5515317\end{array}$ 

C $\quad 3.3766456 \quad 7.2442116 \quad 4.6853122$
C $\quad 4.0689318 \quad 11.2122780 \quad 7.9973027$
C $\quad 3.2665944 \quad 11.7047107 \quad 6.9730854$
C $\quad 5.1610361 \quad 12.0257204 \quad 8.5061681$
$\begin{array}{llll}\text { C } & 9.3873600 & 11.2915271 & 7.1044739\end{array}$
C $\quad 9.8025268 \quad 10.6622138 \quad 5.9247803$
C $\quad 5.4195187 \quad 13.2831585 \quad 7.9483020$
C $\quad 4.5861886 \quad 13.7855187 \quad 6.8682187$
$\begin{array}{llll}\text { C } & 3.5229749 & 13.0131660 & 6.3953697\end{array}$
C $\quad 6.7889817 \quad 13.6903446 \quad 7.6837293$
C $\quad 7.8481212 \quad 12.8309713 \quad 7.9987091$
C $\quad 8.9625330 \quad 12.6813560 \quad 7.0766642$
$\begin{array}{llll}\text { C } & 3.1793000 & 6.5368240 & 3.3218470\end{array}$
$\mathrm{H} \quad 3.7132962 \quad 7.0818830 \quad 2.5328435$
$\mathrm{H} \quad 2.1074613 \quad 6.6128824 \quad 3.0767888$
C $\quad 3.6142650 \quad 5.0682511 \quad 3.3005830$
$\mathrm{H} \quad 4.6756720 \quad 4.9823005 \quad 3.5782068$
$\mathrm{H} \quad 3.0545555 \quad 4.4884207 \quad 4.0474761$
$\begin{array}{llll}\text { C } & 0.2564084 & 6.2643088 & 6.6690647\end{array}$
$\begin{array}{llll}\text { C } & 0.7412981 & 5.2588478 & 7.5096041\end{array}$
C $\quad 1.1192989 \quad 6.9143642 \quad 5.7853708$
H $\quad 0.0699256 \quad 4.7519076 \quad 8.2039153$
$\mathrm{H} \quad 0.7318813 \quad 7.7020540 \quad 5.1353734$
C $\quad 2.0915915 \quad 4.9079201 \quad 7.4546667$
C $\quad 2.4789508 \quad 6.5755983 \quad 5.7282151$
H $\quad 2.4799451 \quad 4.1209041 \quad 8.1026949$
C $\quad 2.9534702 \quad 5.5611263 \quad 6.5695686$
$\mathrm{H} \quad 4.0059783 \quad 5.2761214 \quad 6.5371839$
$\begin{array}{llll}\mathrm{H} & -0.7967540 & 6.5468937 & 6.7030818\end{array}$
C $\quad 3.4069653 \quad 4.4430105 \quad 1.9220101$
H $\quad 2.3517478 \quad 4.5061686 \quad 1.6068311$ 


$$
\begin{array}{llll}
\mathrm{H} & 3.9740647 & 4.9802336 & 1.1436557 \\
\mathrm{C} & 3.8136283 & 2.9870528 & 1.8609819 \\
\mathrm{O} & 4.2572922 & 2.3253343 & 2.7767515 \\
\mathrm{O} & 3.6194093 & 2.4720278 & 0.6097890 \\
\mathrm{H} & 3.9046034 & 1.5355958 & 0.6591805
\end{array}
$$

\section{c. Monomer- $\mathrm{C}_{61}(\mathrm{CN})_{2}$}
65

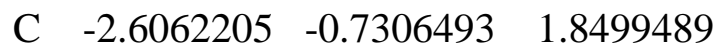
$\begin{array}{llll}\text { C } & -1.4594237 & -1.1956238 & 2.6045227\end{array}$
$\begin{array}{llll}\text { C } & -0.7898046 & 0.0000000 & 3.2058100\end{array}$
$\begin{array}{llll}\text { C } & -1.4594237 & 1.1956238 & 2.6045227\end{array}$
$\begin{array}{llll}\text { C } & -2.6062205 & 0.7306493 & 1.8499489\end{array}$
$\begin{array}{llll}\text { C } & 2.6062205 & -0.7306493 & 1.8499489\end{array}$
C $\quad 2.6062205 \quad 0.7306493 \quad 1.8499489$
$\begin{array}{llll}\text { C } & 1.4594237 & 1.1956238 & 2.6045227\end{array}$
C $\quad 0.7898046 \quad 0.0000000 \quad 3.2058100$
$\begin{array}{llll}\text { C } & 1.4594237 & -1.1956238 & 2.6045227\end{array}$
C $\quad 1.1826091 \quad 3.0287293 \quad 0.9818939$
$\begin{array}{llll}\text { C } & -0.0000000 & 3.4792202 & 0.2518958\end{array}$
C $\quad-1.1826091 \quad 3.0287293 \quad 0.9818939$
$\begin{array}{llll}\text { C } & -0.7412615 & 2.3016879 & 2.1647958\end{array}$
$\begin{array}{llll}\text { C } & 0.7412615 & 2.3016879 & 2.1647958\end{array}$
C $\quad-2.3203281 \quad 2.6131998 \quad-1.1871422$
$\begin{array}{llll}\text { C } & -3.0523375 & 1.4327730 & -1.6429665\end{array}$
$\begin{array}{llll}\text { C } & -3.5067970 & 0.7003288 & -0.4630686\end{array}$
C $\quad-3.0489284 \quad 1.4309656 \quad 0.7175941$
$\begin{array}{llll}\text { C } & -2.3205766 & 2.6074791 & 0.2745894\end{array}$
$\begin{array}{llll}\text { C } & -3.0523375 & -1.4327730 & -1.6429665\end{array}$ 

C $\quad-2.3203281 \quad-2.6131998 \quad-1.1871422$
$\begin{array}{llll}\text { C } & -2.3205766 & -2.6074791 & 0.2745894\end{array}$
C $\quad-3.0489284 \quad-1.4309656 \quad 0.7175941$
$\begin{array}{llll}\text { C } & -3.5067970 & -0.7003288 & -0.4630686\end{array}$
$\begin{array}{llll}\text { C } & -0.0000000 & -3.4792202 & 0.2518958\end{array}$
C $\quad \begin{array}{lll}1.1826091 & -3.0287293 & 0.9818939\end{array}$
$\begin{array}{llll}\text { C } & 0.7412615 & -2.3016879 & 2.1647958\end{array}$
$\begin{array}{llll}\text { C } & -0.7412615 & -2.3016879 & 2.1647958\end{array}$
C $\quad-1.1826091 \quad-3.0287293 \quad 0.9818939$
$\begin{array}{llll}\text { C } & 2.3203281 & -2.6131998 & -1.1871422\end{array}$
C $\quad 3.0523375 \quad-1.4327730 \quad-1.6429665$
$\begin{array}{llll}\text { C } & 3.5067970 & -0.7003288 & -0.4630686\end{array}$
C $\quad 3.0489284 \quad-1.4309656 \quad 0.7175941$
$\begin{array}{llll}\text { C } & 2.3205766 & -2.6074791 & 0.2745894\end{array}$
C $\quad 3.5067970 \quad 0.7003288 \quad-0.4630686$
C $\quad 3.0523375 \quad 1.4327730 \quad-1.6429665$
C $\quad 2.3203281 \quad 2.6131998 \quad-1.1871422$
$\begin{array}{llll}\text { C } & 2.3205766 & 2.6074791 & 0.2745894\end{array}$
$\begin{array}{llll}\text { C } & 3.0489284 & 1.4309656 & 0.7175941\end{array}$
C $\quad \begin{array}{llll}1.1828080 & 3.0424828 & -1.8841240\end{array}$
C $\quad 0.7320418 \quad 2.3158185 \quad-3.0657890$
$\begin{array}{llll}\text { C } & -0.7320418 & 2.3158185 & -3.0657890\end{array}$
C $\quad-1.1828080 \quad 3.0424828 \quad-1.8841240$
$\begin{array}{llll}\text { C } & -0.0000000 & 3.4904192 & -1.1489532\end{array}$
$\begin{array}{llll}\text { C } & -1.4324334 & 1.1819899 & -3.5017671\end{array}$
$\begin{array}{llll}\text { C } & -0.7007999 & 0.0000000 & -3.9543357\end{array}$
C $-1.4324334-1.1819899 \quad-3.5017671$
C $-2.6168656-0.7316586-2.7761675$
C $\quad-2.6168656 \quad 0.7316586-2.7761675$
C $\quad-0.7320418-2.3158185 \quad-3.0657890$
$\begin{array}{llll}\text { C } & 0.7320418 & -2.3158185 & -3.0657890\end{array}$ 


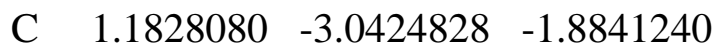
C $\quad-0.0000000 \quad-3.4904192 \quad-1.1489532$
C $-1.1828080-3.0424828-1.8841240$
$\begin{array}{llll}\text { C } & 1.4324334 & -1.1819899 & -3.5017671\end{array}$
$\begin{array}{llll}\text { C } & 0.7007999 & 0.0000000 & -3.9543357\end{array}$
$\begin{array}{llll}\text { C } & 1.4324334 & 1.1819899 & -3.5017671\end{array}$
C $\quad 2.6168656 \quad 0.7316586 \quad-2.7761675$
C $\quad 2.6168656-0.7316586 \quad-2.7761675$
$\begin{array}{llll}\text { C } & -0.0000000 & 0.0000000 & 4.5568463\end{array}$
$\begin{array}{llll}\text { C } & 0.0000000 & -1.2240657 & 5.3183368\end{array}$
$\begin{array}{llll}\text { C } & 0.0000000 & 1.2240657 & 5.3183368\end{array}$
N $\quad 0.0000000 \quad-2.2419162 \quad 5.9041836$
$\mathrm{N} \quad 0.0000000 \quad 2.2419162 \quad 5.9041836$

\section{d. $1 \mathrm{D}_{2 \mathrm{~h}}$}

130
$\begin{array}{llll}\text { C } & -2.6061400 & 0.7306322 & -7.3532334\end{array}$
$\begin{array}{llll}\text { C } & -1.4593554 & 1.1955637 & -8.1078764\end{array}$
$\begin{array}{llll}\text { C } & -0.7898274 & 0.0000000 & -8.7093530\end{array}$

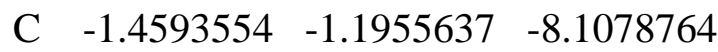
C $\quad-2.6061400 \quad-0.7306322 \quad-7.3532334$
$\begin{array}{llll}\text { C } & 2.6061400 & 0.7306322 & -7.3532334\end{array}$
$\begin{array}{llll}\text { C } & 2.6061400 & -0.7306322 & -7.3532334\end{array}$
$\begin{array}{llll}\text { C } & 1.4593554 & -1.1955637 & -8.1078764\end{array}$
C $\quad 0.7898274 \quad 0.0000000 \quad-8.7093530$
$\begin{array}{llll}\text { C } & 1.4593554 & 1.1955637 & -8.1078764\end{array}$
$\begin{array}{llll}\text { C } & 1.1825644 & -3.0286879 & -6.4852414\end{array}$
C $\quad 0.0000000 \quad-3.4795515 \quad-5.7553976$
$\begin{array}{llll}\text { C } & -1.1825644 & -3.0286879 & -6.4852414\end{array}$ 

C $\quad-0.7412437 \quad-2.3016015 \quad-7.6681632$
C $\quad 0.7412437 \quad-2.3016015 \quad-7.6681632$
C $\quad-2.3205098 \quad-2.6133920 \quad-4.3162402$
C $\quad-3.0535641-1.4330636 \quad-3.8604786$
C $\quad-3.5073982-0.7003907 \quad-5.0405679$
C $\quad-3.0490593 \quad-1.4310129-6.2210564$
$\begin{array}{llll}\text { C } & -2.3205417 & -2.6074224 & -5.7779186\end{array}$
$\begin{array}{llll}\text { C } & -3.0535641 & 1.4330636 & -3.8604786\end{array}$
$\begin{array}{lllll}\text { C } & -2.3205098 & 2.6133920 & -4.3162402\end{array}$
$\begin{array}{llll}\text { C } & -2.3205417 & 2.6074224 & -5.7779186\end{array}$
$\begin{array}{llll}\text { C } & -3.0490593 & 1.4310129 & -6.2210564\end{array}$
$\begin{array}{llll}\text { C } & -3.5073982 & 0.7003907 & -5.0405679\end{array}$
$\begin{array}{llll}\text { C } & 0.0000000 & 3.4795515 & -5.7553976\end{array}$
$\begin{array}{llll}\text { C } & 1.1825644 & 3.0286879 & -6.4852414\end{array}$
$\begin{array}{llll}\text { C } & 0.7412437 & 2.3016015 & -7.6681632\end{array}$
$\begin{array}{llll}\text { C } & -0.7412437 & 2.3016015 & -7.6681632\end{array}$
$\begin{array}{llll}\text { C } & -1.1825644 & 3.0286879 & -6.4852414\end{array}$
$\begin{array}{llll}\text { C } & 2.3205098 & 2.6133920 & -4.3162402\end{array}$
C $3.0535641 \quad 1.4330636 \quad-3.8604786$
$\begin{array}{llll}\text { C } & 3.5073982 & 0.7003907 & -5.0405679\end{array}$
$\begin{array}{llll}\text { C } & 3.0490593 & 1.4310129 & -6.2210564\end{array}$
$\begin{array}{llll}\text { C } & 2.3205417 & 2.6074224 & -5.7779186\end{array}$
$\begin{array}{llll}\text { C } & 3.5073982 & -0.7003907 & -5.0405679\end{array}$
C $3.0535641-1.4330636 \quad-3.8604786$
$\begin{array}{llll}\text { C } & 2.3205098 & -2.6133920 & -4.3162402\end{array}$
$\begin{array}{llll}\text { C } & 2.3205417 & -2.6074224 & -5.7779186\end{array}$
C $3.0490593 \quad-1.4310129 \quad-6.2210564$
$\begin{array}{llll}\text { C } & 1.1830999 & -3.0437263 & -3.6191458\end{array}$
C $\quad 0.7321528 \quad-2.3170527 \quad-2.4377821$
C $\quad-0.7321528 \quad-2.3170527 \quad-2.4377821$
$\begin{array}{llll}\text { C } & -1.1830999 & -3.0437263 & -3.6191458\end{array}$ 


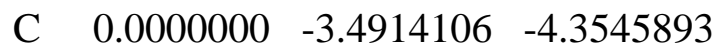
$\begin{array}{llll}\text { C } & -1.4325909 & -1.1820480 & -2.0037214\end{array}$
$\begin{array}{llll}\text { C } & -0.6998645 & 0.0000000 & -1.5616336\end{array}$
$\begin{array}{llll}\text { C } & -1.4325909 & 1.1820480 & -2.0037214\end{array}$
$\begin{array}{llll}\text { C } & -2.6188811 & 0.7321517 & -2.7270597\end{array}$
C $\quad-2.6188811 \quad-0.7321517 \quad-2.7270597$
$\begin{array}{llll}\text { C } & -0.7321528 & 2.3170527 & -2.4377821\end{array}$
$\begin{array}{llll}\text { C } & 0.7321528 & 2.3170527 & -2.4377821\end{array}$
$\begin{array}{llll}\text { C } & 1.1830999 & 3.0437263 & -3.6191458\end{array}$
C $\quad 0.0000000 \quad 3.4914106 \quad-4.3545893$
$\begin{array}{llll}\text { C } & -1.1830999 & 3.0437263 & -3.6191458\end{array}$
$\begin{array}{llll}\text { C } & 1.4325909 & 1.1820480 & -2.0037214\end{array}$
C $\quad 0.6998645 \quad 0.0000000 \quad-1.5616336$
$\begin{array}{llll}\text { C } & 1.4325909 & -1.1820480 & -2.0037214\end{array}$
$\begin{array}{llll}\text { C } & 2.6188811 & -0.7321517 & -2.7270597\end{array}$
$\begin{array}{llll}\text { C } & 2.6188811 & 0.7321517 & -2.7270597\end{array}$
C $\quad 0.0000000 \quad 0.0000000-10.0602526$
C $\quad-0.0000000 \quad 1.2241144 \quad-10.8217382$
C $\quad-0.0000000 \quad-1.2241144-10.8217382$
N $\quad-0.0000000 \quad 2.2419892-11.4075073$
$\mathrm{N} \quad-0.0000000 \quad-2.2419892-11.4075073$
$\begin{array}{llll}\text { C } & -2.6061400 & -0.7306322 & 7.3532334\end{array}$
C $\quad-1.4593554-1.1955637 \quad 8.1078764$
$\begin{array}{llll}\text { C } & -0.7898274 & 0.0000000 & 8.7093530\end{array}$

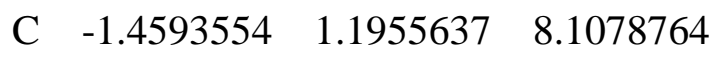
$\begin{array}{llll}\text { C } & -2.6061400 & 0.7306322 & 7.3532334\end{array}$
$\begin{array}{llll}\text { C } & 2.6061400 & -0.7306322 & 7.3532334\end{array}$
$\begin{array}{llll}\text { C } & 2.6061400 & 0.7306322 & 7.3532334\end{array}$
C $\quad 1.4593554 \quad 1.1955637 \quad 8.1078764$
C $\quad 0.7898274 \quad 0.0000000 \quad 8.7093530$
$\begin{array}{llll}\text { C } & 1.4593554 & -1.1955637 & 8.1078764\end{array}$ 

C $\quad 1.1825644 \quad 3.0286879 \quad 6.4852414$
C $\quad 0.0000000 \quad 3.4795515 \quad 5.7553976$
$\begin{array}{llll}\text { C } & -1.1825644 & 3.0286879 & 6.4852414\end{array}$
$\begin{array}{llll}\text { C } & -0.7412437 & 2.3016015 & 7.6681632\end{array}$
C $\quad 0.7412437 \quad 2.3016015 \quad 7.6681632$
$\begin{array}{llll}\text { C } & -2.3205098 & 2.6133920 & 4.3162402\end{array}$
$\begin{array}{llll}\text { C } & -3.0535641 & 1.4330636 & 3.8604786\end{array}$
$\begin{array}{llll}\text { C } & -3.5073982 & 0.7003907 & 5.0405679\end{array}$
$\begin{array}{llll}\text { C } & -3.0490593 & 1.4310129 & 6.2210564\end{array}$
$\begin{array}{llll}\text { C } & -2.3205417 & 2.6074224 & 5.7779186\end{array}$
C $\quad-3.0535641 \quad-1.4330636 \quad 3.8604786$
C $\quad-2.3205098 \quad-2.6133920 \quad 4.3162402$
$\begin{array}{llll}\text { C } & -2.3205417 & -2.6074224 & 5.7779186\end{array}$
$\begin{array}{llll}\text { C } & -3.0490593 & -1.4310129 & 6.2210564\end{array}$
C $\quad-3.5073982 \quad-0.7003907 \quad 5.0405679$
C $\quad 0.0000000 \quad-3.4795515 \quad 5.7553976$
$\begin{array}{llll}\text { C } & 1.1825644 & -3.0286879 & 6.4852414\end{array}$
$\begin{array}{llll}\text { C } & 0.7412437 & -2.3016015 & 7.6681632\end{array}$
C $\quad-0.7412437 \quad-2.3016015 \quad 7.6681632$
C $\quad-1.1825644 \quad-3.0286879 \quad 6.4852414$
C $\quad 2.3205098 \quad-2.6133920 \quad 4.3162402$
C $\quad 3.0535641 \quad-1.4330636 \quad 3.8604786$
$\begin{array}{llll}\text { C } & 3.5073982 & -0.7003907 & 5.0405679\end{array}$
$\begin{array}{llll}\text { C } & 3.0490593 & -1.4310129 & 6.2210564\end{array}$
$\begin{array}{llll}\text { C } & 2.3205417 & -2.6074224 & 5.7779186\end{array}$
C $\quad 3.5073982 \quad 0.7003907 \quad 5.0405679$
C $\quad 3.0535641 \quad 1.4330636 \quad 3.8604786$
C $\quad 2.3205098 \quad 2.6133920 \quad 4.3162402$
C $\quad 2.3205417 \quad 2.6074224 \quad 5.7779186$
C $\quad 3.0490593 \quad 1.4310129 \quad 6.2210564$
$\begin{array}{llll}\text { C } & 1.1830999 & 3.0437263 & 3.6191458\end{array}$ 

C $\quad 0.7321528 \quad 2.3170527 \quad 2.4377821$
$\begin{array}{llll}\text { C } & -0.7321528 & 2.3170527 & 2.4377821\end{array}$
C $\quad-1.1830999 \quad 3.0437263 \quad 3.6191458$
C $\quad 0.0000000 \quad 3.4914106 \quad 4.3545893$
$\begin{array}{llll}\text { C } & -1.4325909 & 1.1820480 & 2.0037214\end{array}$
$\begin{array}{llll}\text { C } & -0.6998645 & 0.0000000 & 1.5616336\end{array}$
$\begin{array}{llll}\text { C } & -1.4325909 & -1.1820480 & 2.0037214\end{array}$
$\begin{array}{llll}\text { C } & -2.6188811 & -0.7321517 & 2.7270597\end{array}$
$\begin{array}{llll}\text { C } & -2.6188811 & 0.7321517 & 2.7270597\end{array}$
$\begin{array}{llll}\text { C } & -0.7321528 & -2.3170527 & 2.4377821\end{array}$
$\begin{array}{llll}\text { C } & 0.7321528 & -2.3170527 & 2.4377821\end{array}$
C $\quad 1.1830999 \quad-3.0437263 \quad 3.6191458$
C $\quad 0.0000000 \quad-3.4914106 \quad 4.3545893$
$\begin{array}{llll}\text { C } & -1.1830999 & -3.0437263 & 3.6191458\end{array}$
$\begin{array}{llll}\text { C } & 1.4325909 & -1.1820480 & 2.0037214\end{array}$
C $\quad 0.6998645 \quad 0.0000000 \quad 1.5616336$
C $\quad 1.4325909 \quad 1.1820480 \quad 2.0037214$
$\begin{array}{llll}\text { C } & 2.6188811 & 0.7321517 & 2.7270597\end{array}$
$\begin{array}{llll}\text { C } & 2.6188811 & -0.7321517 & 2.7270597\end{array}$
C $\quad 0.0000000 \quad 0.0000000 \quad 10.0602526$
C $\quad-0.0000000 \quad-1.2241144 \quad 10.8217382$
$\begin{array}{llll}\text { C } & -0.0000000 & 1.2241144 & 10.8217382\end{array}$
$\begin{array}{llll}\mathrm{N} & -0.0000000 & -2.2419892 & 11.4075073\end{array}$
$\begin{array}{llll}\mathrm{N} & -0.0000000 & 2.2419892 & 11.4075073\end{array}$

\section{e. $2 D_{2 d}$}

130

C $\quad-2.3591575 \quad-1.3266877 \quad-7.3283408$

$\begin{array}{llll}\text { C } & -1.8779411 & -0.1865470 & -8.0841235\end{array}$ 


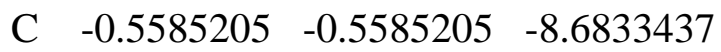
$\begin{array}{llll}\text { C } & -0.1865470 & -1.8779411 & -8.0841235\end{array}$
C $\quad-1.3266877 \quad-2.3591575 \quad-7.3283408$
$\begin{array}{llll}\text { C } & 1.3266877 & 2.3591575 & -7.3283408\end{array}$
$\begin{array}{llll}\text { C } & 2.3591575 & 1.3266877 & -7.3283408\end{array}$
$\begin{array}{llll}\text { C } & 1.8779411 & 0.1865470 & -8.0841235\end{array}$
$\begin{array}{llll}\text { C } & 0.5585205 & 0.5585205 & -8.6833437\end{array}$
C $\quad 0.1865470 \quad 1.8779411 \quad-8.0841235$
C $\quad 2.9777287 \quad-1.3052159 \quad-6.4603930$
$\begin{array}{llll}\text { C } & 2.4594009 & -2.4594009 & -5.7309334\end{array}$
C $\quad \begin{array}{llll}1.3052159 & -2.9777287 & -6.4603930\end{array}$
C $\quad \begin{array}{lll}1.1035985 & -2.1512633 & -7.6438855\end{array}$
C $\quad 2.1512633 \quad-1.1035985 \quad-7.6438855$
C $\quad 0.2071241 \quad-3.4889663 \quad-4.2918333$
C $\quad-1.1463085-3.1729710 \quad-3.8355992$
$\begin{array}{llll}\text { C } & -1.9839329 & -2.9742623 & -5.0158847\end{array}$
C $-1.1444001-3.1678258-6.1962419$
$\begin{array}{llll}\text { C } & 0.2028717 & -3.4849857 & -5.7527168\end{array}$

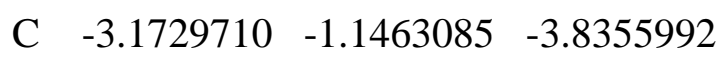
C $\quad-3.4889663 \quad 0.2071241 \quad-4.2918333$
$\begin{array}{llll}\text { C } & -3.4849857 & 0.2028717 & -5.7527168\end{array}$
C $\quad-3.1678258-1.1444001 \quad-6.1962419$
$\begin{array}{llll}\text { C } & -2.9742623 & -1.9839329 & -5.0158847\end{array}$
$\begin{array}{llll}\text { C } & -2.4594009 & 2.4594009 & -5.7309334\end{array}$
C $\quad-1.3052159 \quad 2.9777287 \quad-6.4603930$
C $\quad-1.1035985 \quad 2.1512633 \quad-7.6438855$

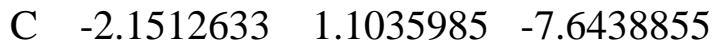
C $\quad-2.9777287 \quad 1.3052159 \quad-6.4603930$
C $\quad-0.2071241 \quad 3.4889663 \quad-4.2918333$
$\begin{array}{llll}\text { C } & 1.1463085 & 3.1729710 & -3.8355992\end{array}$
$\begin{array}{llll}\text { C } & 1.9839329 & 2.9742623 & -5.0158847\end{array}$ 

C $\quad 1.1444001 \quad 3.1678258 \quad-6.1962419$
$\begin{array}{llll}\text { C } & -0.2028717 & 3.4849857 & -5.7527168\end{array}$
$\begin{array}{llll}\text { C } & 2.9742623 & 1.9839329 & -5.0158847\end{array}$

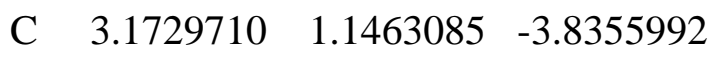
C $3.4889663 \quad-0.2071241 \quad-4.2918333$
$\begin{array}{llll}\text { C } & 3.4849857 & -0.2028717 & -5.7527168\end{array}$
$\begin{array}{llll}\text { C } & 3.1678258 & 1.1444001 & -6.1962419\end{array}$
$\begin{array}{llll}\text { C } & 2.9892761 & -1.3158509 & -3.5945264\end{array}$
C $\quad 2.1559702 \quad-1.1211635 \quad-2.4126223$
C $\quad \begin{array}{llll}1.1211635 & -2.1559702 & -2.4126223\end{array}$
$\begin{array}{llll}\text { C } & 1.3158509 & -2.9892761 & -3.5945264\end{array}$
C $\quad 2.4679767 \quad-2.4679767 \quad-4.3295320$
$\begin{array}{llll}\text { C } & -0.1768278 & -1.8490361 & -1.9786573\end{array}$
C $\quad-0.4948241 \quad-0.4948241 \quad-1.5400322$
C $\quad-1.8490361 \quad-0.1768278-1.9786573$
C $\quad-2.3694306-1.3347800 \quad-2.7027638$
C $\quad-1.3347800 \quad-2.3694306 \quad-2.7027638$
$\begin{array}{llll}\text { C } & -2.1559702 & 1.1211635 & -2.4126223\end{array}$
C $\quad-1.1211635 \quad 2.1559702 \quad-2.4126223$
$\begin{array}{llll}\text { C } & -1.3158509 & 2.9892761 & -3.5945264\end{array}$
$\begin{array}{llll}\text { C } & -2.4679767 & 2.4679767 & -4.3295320\end{array}$
$\begin{array}{llll}\text { C } & -2.9892761 & 1.3158509 & -3.5945264\end{array}$
$\begin{array}{lllll}\text { C } & 0.1768278 & 1.8490361 & -1.9786573\end{array}$
$\begin{array}{llll}\text { C } & 0.4948241 & 0.4948241 & -1.5400322\end{array}$
C $\quad \begin{array}{llll}1.8490361 & 0.1768278 & -1.9786573\end{array}$
$\begin{array}{llll}\text { C } & 2.3694306 & 1.3347800 & -2.7027638\end{array}$
$\begin{array}{llll}\text { C } & 1.3347800 & 2.3694306 & -2.7027638\end{array}$
C $\quad 0.0000000 \quad 0.0000000-10.0357319$
$\begin{array}{llll}\text { C } & -0.8658047 & 0.8658047 & -10.7963881\end{array}$
$\begin{array}{llll}\text { C } & 0.8658047 & -0.8658047 & -10.7963881\end{array}$
$\mathrm{N} \quad-1.5863946 \quad 1.5863946-11.3802187$ 


$$
\begin{array}{lrrr}
\mathrm{N} & 1.5863946 & -1.5863946 & -11.3802187 \\
\mathrm{C} & -2.3591575 & 1.3266877 & 7.3283408 \\
\mathrm{C} & -1.8779411 & 0.1865470 & 8.0841235 \\
\mathrm{C} & -0.5585205 & 0.5585205 & 8.6833437 \\
\mathrm{C} & -0.1865470 & 1.8779411 & 8.0841235 \\
\mathrm{C} & -1.3266877 & 2.3591575 & 7.3283408 \\
\mathrm{C} & 1.3266877 & -2.3591575 & 7.3283408 \\
\mathrm{C} & 2.3591575 & -1.3266877 & 7.3283408 \\
\mathrm{C} & 1.8779411 & -0.1865470 & 8.0841235 \\
\mathrm{C} & 0.5585205 & -0.5585205 & 8.6833437 \\
\mathrm{C} & 0.1865470 & -1.8779411 & 8.0841235 \\
\mathrm{C} & 2.9777287 & 1.3052159 & 6.4603930 \\
\mathrm{C} & 2.4594009 & 2.4594009 & 5.7309334 \\
\mathrm{C} & 1.3052159 & 2.9777287 & 6.4603930 \\
\mathrm{C} & 1.1035985 & 2.1512633 & 7.6438855 \\
\mathrm{C} & 2.1512633 & 1.1035985 & 7.6438855 \\
\mathrm{C} & 0.2071241 & 3.4889663 & 4.2918333 \\
\mathrm{C} & -1.1463085 & 3.1729710 & 3.8355992 \\
\mathrm{C} & -1.9839329 & 2.9742623 & 5.0158847 \\
\mathrm{C} & -1.1444001 & 3.1678258 & 6.1962419 \\
\mathrm{C} & 0.2028717 & 3.4849857 & 5.7527168 \\
\mathrm{C} & -3.1729710 & 1.1463085 & 3.8355992 \\
\mathrm{C} & -3.4889663 & -0.2071241 & 4.2918333 \\
\mathrm{C} & -3.4849857 & -0.2028717 & 5.7527168 \\
\mathrm{C} & -3.1678258 & 1.1444001 & 6.1962419 \\
\mathrm{C} & -2.9742623 & 1.9839329 & 5.0158847 \\
\mathrm{C} & -2.4594009 & -2.4594009 & 5.7309334 \\
\mathrm{C} & -1.3052159 & -2.9777287 & 6.4603930 \\
\mathrm{C} & -1.1035985 & -2.1512633 & 7.6438855 \\
\hline & -2.1512633 & -1.1035985 & 7.6438855 \\
\hline
\end{array}
$$



C $\quad-0.2071241 \quad-3.4889663 \quad 4.2918333$
C $\quad 1.1463085 \quad-3.1729710 \quad 3.8355992$
$\begin{array}{llll}\text { C } & 1.9839329 & -2.9742623 & 5.0158847\end{array}$
C $\quad 1.1444001 \quad-3.1678258 \quad 6.1962419$
$\begin{array}{llll}\text { C } & -0.2028717 & -3.4849857 & 5.7527168\end{array}$
$\begin{array}{llll}\text { C } & 2.9742623 & -1.9839329 & 5.0158847\end{array}$
C $\quad 3.1729710 \quad-1.1463085 \quad 3.8355992$
$\begin{array}{llll}\text { C } & 3.4889663 & 0.2071241 & 4.2918333\end{array}$
$\begin{array}{llll}\text { C } & 3.4849857 & 0.2028717 & 5.7527168\end{array}$
C $\quad 3.1678258 \quad-1.1444001 \quad 6.1962419$
$\begin{array}{llll}\text { C } & 2.9892761 & 1.3158509 & 3.5945264\end{array}$
C $\quad 2.1559702 \quad 1.1211635 \quad 2.4126223$
C $\quad 1.1211635 \quad 2.1559702 \quad 2.4126223$
C $\quad 1.3158509 \quad 2.9892761 \quad 3.5945264$
C $\quad 2.4679767 \quad 2.4679767 \quad 4.3295320$
$\begin{array}{llll}\text { C } & -0.1768278 & 1.8490361 & 1.9786573\end{array}$
$\begin{array}{llll}\text { C } & -0.4948241 & 0.4948241 & 1.5400322\end{array}$
$\begin{array}{llll}\text { C } & -1.8490361 & 0.1768278 & 1.9786573\end{array}$
$\begin{array}{llll}\text { C } & -2.3694306 & 1.3347800 & 2.7027638\end{array}$
$\begin{array}{llll}\text { C } & -1.3347800 & 2.3694306 & 2.7027638\end{array}$
C $\quad-2.1559702-1.1211635 \quad 2.4126223$
$\begin{array}{llll}\text { C } & -1.1211635 & -2.1559702 & 2.4126223\end{array}$
C $\quad-1.3158509 \quad-2.9892761 \quad 3.5945264$
C $\quad-2.4679767-2.4679767 \quad 4.3295320$
C $\quad-2.9892761 \quad-1.3158509 \quad 3.5945264$
C $\quad 0.1768278 \quad-1.8490361 \quad 1.9786573$
$\begin{array}{llll}\text { C } & 0.4948241 & -0.4948241 & 1.5400322\end{array}$
$\begin{array}{llll}\text { C } & 1.8490361 & -0.1768278 & 1.9786573\end{array}$
$\begin{array}{llll}\text { C } & 2.3694306 & -1.3347800 & 2.7027638\end{array}$
$\begin{array}{llll}\text { C } & 1.3347800 & -2.3694306 & 2.7027638\end{array}$
$\begin{array}{llll}\text { C } & 0.0000000 & 0.0000000 & 10.0357319\end{array}$ 

C $\quad-0.8658047 \quad-0.8658047 \quad 10.7963881$
$\begin{array}{llll}\text { C } & 0.8658047 & 0.8658047 & 10.7963881\end{array}$
N $\quad-1.5863946 \quad-1.5863946 \quad 11.3802187$
$\begin{array}{llll}\mathrm{N} & 1.5863946 & 1.5863946 & 11.3802187\end{array}$

\section{f. $3 \mathrm{C}_{2 \mathrm{v}}$}

130
$\begin{array}{llll}\text { C } & -7.3559284 & -0.7304697 & 1.8875618\end{array}$
$\begin{array}{llll}\text { C } & -6.1897919 & -1.1957842 & 2.6123755\end{array}$
$\begin{array}{llll}\text { C } & -5.5050703 & 0.0000000 & 3.1958430\end{array}$
$\begin{array}{llll}\text { C } & -6.1897919 & 1.1957842 & 2.6123755\end{array}$
$\begin{array}{llll}\text { C } & -7.3559284 & 0.7304697 & 1.8875618\end{array}$
$\begin{array}{llll}\text { C } & -2.1475845 & -0.7306387 & 1.7523289\end{array}$
$\begin{array}{llll}\text { C } & -2.1475845 & 0.7306387 & 1.7523289\end{array}$
$\begin{array}{llll}\text { C } & -3.2723576 & 1.1960557 & 2.5370936\end{array}$
$\begin{array}{llll}\text { C } & -3.9260730 & 0.0000000 & 3.1561652\end{array}$
C $\quad-3.2723576-1.1960557 \quad 2.5370936$
$\begin{array}{llll}\text { C } & -3.5908189 & 3.0310915 & 0.9215144\end{array}$
$\begin{array}{llll}\text { C } & -4.7925005 & 3.4800543 & 0.2222366\end{array}$
$\begin{array}{llll}\text { C } & -5.9554386 & 3.0289813 & 0.9827799\end{array}$
C $\quad \begin{array}{llll}-5.4831211 & 2.3018118 & 2.1538559\end{array}$
C $\quad-4.0013634 \quad 2.3027152 \quad 2.1155495$
$\begin{array}{llll}\text { C } & -7.1488022 & 2.6127104 & -1.1558634\end{array}$
$\begin{array}{llll}\text { C } & -7.8928904 & 1.4327045 & -1.5925791\end{array}$
$\begin{array}{llll}\text { C } & -8.3164023 & 0.7003328 & -0.4010951\end{array}$
$\begin{array}{llll}\text { C } & -7.8278171 & 1.4308038 & 0.7670234\end{array}$
C $\quad \begin{array}{llll}-7.1113517 & 2.6076679 & 0.3053113\end{array}$
$\begin{array}{llll}\text { C } & -7.8928904 & -1.4327045 & -1.5925791\end{array}$
$\begin{array}{llll}\text { C } & -7.1488022 & -2.6127104 & -1.1558634\end{array}$ 

C $\quad-7.1113517 \quad-2.6076679 \quad 0.3053113$
C $\quad-7.8278171-1.4308038 \quad 0.7670234$
C $\quad-8.3164023 \quad-0.7003328 \quad-0.4010951$
$\begin{array}{llll}\text { C } & -4.7925005 & -3.4800543 & 0.2222366\end{array}$
$\begin{array}{llll}\text { C } & -3.5908189 & -3.0310915 & 0.9215144\end{array}$
C $\quad-4.0013634-2.3027152 \quad 2.1155495$
C $\quad-5.4831211 \quad-2.3018118 \quad 2.1538559$
C $\quad-5.9554386 \quad-3.0289813 \quad 0.9827799$
C $\quad-2.5097027 \quad-2.6167523 \quad-1.2774843$
C $\quad-1.7944372-1.4321444 \quad-1.7507004$
$\begin{array}{llll}\text { C } & -1.3201491 & -0.7001077 & -0.5841434\end{array}$
$\begin{array}{llll}\text { C } & -1.7364174 & -1.4306447 & 0.6073708\end{array}$
$\begin{array}{llll}\text { C } & -2.4716898 & -2.6105800 & 0.1846454\end{array}$
$\begin{array}{llll}\text { C } & -1.3201491 & 0.7001077 & -0.5841434\end{array}$
C $\quad-1.7944372 \quad 1.4321444 \quad-1.7507004$
$\begin{array}{llll}\text { C } & -2.5097027 & 2.6167523 & -1.2774843\end{array}$
$\begin{array}{llll}\text { C } & -2.4716898 & 2.6105800 & 0.1846454\end{array}$
$\begin{array}{llll}\text { C } & -1.7364174 & 1.4306447 & 0.6073708\end{array}$
$\begin{array}{llll}\text { C } & -3.6656155 & 3.0443094 & -1.9443776\end{array}$
$\begin{array}{llll}\text { C } & -4.1469933 & 2.3161629 & -3.1141089\end{array}$
$\begin{array}{llll}\text { C } & -5.6103999 & 2.3155127 & -3.0751784\end{array}$
$\begin{array}{llll}\text { C } & -6.0303739 & 3.0423854 & -1.8823763\end{array}$
C $\quad-4.8289158 \quad 3.4912492 \quad-1.1784376$
$\begin{array}{llll}\text { C } & -6.3221469 & 1.1819287 & -3.4929780\end{array}$
$\begin{array}{llll}\text { C } & -5.6028490 & 0.0000000 & -3.9652691\end{array}$
C $\quad-6.3221469-1.1819287 \quad-3.4929780$
$\begin{array}{llll}\text { C } & -7.4872806 & -0.7317249 & -2.7366513\end{array}$
$\begin{array}{llll}\text { C } & -7.4872806 & 0.7317249 & -2.7366513\end{array}$
$\begin{array}{llll}\text { C } & -5.6103999 & -2.3155127 & -3.0751784\end{array}$
$\begin{array}{llll}\text { C } & -4.1469933 & -2.3161629 & -3.1141089\end{array}$
$\begin{array}{llll}\text { C } & -3.6656155 & -3.0443094 & -1.9443776\end{array}$ 

C $\quad-4.8289158-3.4912492 \quad-1.1784376$
C $\quad-6.0303739-3.0423854 \quad-1.8823763$
C $\quad-3.4582546-1.1822011 \quad-3.5694123$
$\begin{array}{llll}\text { C } & -4.2018034 & 0.0000000 & -4.0025320\end{array}$
C $\quad-3.4582546 \quad 1.1822011 \quad-3.5694123$
$\begin{array}{llll}\text { C } & -2.2556095 & 0.7311439 & -2.8754324\end{array}$
$\begin{array}{llll}\text { C } & -2.2556095 & -0.7311439 & -2.8754324\end{array}$
$\begin{array}{llll}\text { C } & -4.6808533 & 0.0000000 & 4.5264119\end{array}$
C $\quad-4.6622955 \quad-1.2245312 \quad 5.2870086$
$\begin{array}{llll}\text { C } & -4.6622955 & 1.2245312 & 5.2870086\end{array}$
$\begin{array}{llll}\mathrm{N} & -4.6492617 & -2.2436062 & 5.8705262\end{array}$
$\begin{array}{llll}\mathrm{N} & -4.6492617 & 2.2436062 & 5.8705262\end{array}$
$\begin{array}{llll}\text { C } & 2.1475845 & -0.7306387 & 1.7523289\end{array}$
C $\quad 3.2723576-1.1960557 \quad 2.5370936$
C $\quad 3.9260730 \quad 0.0000000 \quad 3.1561652$
C $\quad 3.2723576 \quad 1.1960557 \quad 2.5370936$
$\begin{array}{llll}\text { C } & 2.1475845 & 0.7306387 & 1.7523289\end{array}$
$\begin{array}{llll}\text { C } & 7.3559284 & -0.7304697 & 1.8875618\end{array}$
$\begin{array}{llll}\text { C } & 7.3559284 & 0.7304697 & 1.8875618\end{array}$
$\begin{array}{llll}\text { C } & 6.1897919 & 1.1957842 & 2.6123755\end{array}$
C $\quad 5.5050703 \quad 0.0000000 \quad 3.1958430$
$\begin{array}{llll}\text { C } & 6.1897919 & -1.1957842 & 2.6123755\end{array}$
C $\quad \begin{array}{llll}5.9554386 & 3.0289813 & 0.9827799\end{array}$
C $\quad 4.7925005 \quad 3.4800543 \quad 0.2222366$
C $\quad 3.5908189 \quad 3.0310915 \quad 0.9215144$
C $\quad 4.0013634 \quad 2.3027152 \quad 2.1155495$
$\begin{array}{llll}\text { C } & 5.4831211 & 2.3018118 & 2.1538559\end{array}$
C $\quad 2.5097027 \quad 2.6167523 \quad-1.2774843$
C $\quad 1.7944372 \quad 1.4321444 \quad-1.7507004$
$\begin{array}{llll}\text { C } & 1.3201491 & 0.7001077 & -0.5841434\end{array}$
$\begin{array}{llll}\text { C } & 1.7364174 & 1.4306447 & 0.6073708\end{array}$ 

C $\quad 2.4716898 \quad 2.6105800 \quad 0.1846454$
C $\quad \begin{array}{llll}1.7944372 & -1.4321444 & -1.7507004\end{array}$
C $\quad 2.5097027 \quad-2.6167523 \quad-1.2774843$
$\begin{array}{llll}\text { C } & 2.4716898 & -2.6105800 & 0.1846454\end{array}$
$\begin{array}{llll}\text { C } & 1.7364174 & -1.4306447 & 0.6073708\end{array}$
$\begin{array}{llll}\text { C } & 1.3201491 & -0.7001077 & -0.5841434\end{array}$
C $\quad 4.7925005 \quad-3.4800543 \quad 0.2222366$
C $\quad 5.9554386 \quad-3.0289813 \quad 0.9827799$
C $\quad \begin{array}{llll}5.4831211 & -2.3018118 & 2.1538559\end{array}$
C $\quad 4.0013634 \quad-2.3027152 \quad 2.1155495$
C $\quad 3.5908189-3.0310915 \quad 0.9215144$
C $\quad 7.1488022-2.6127104-1.1558634$
C $7.8928904 \quad-1.4327045 \quad-1.5925791$
C $\quad 8.3164023 \quad-0.7003328 \quad-0.4010951$
$\begin{array}{llll}\text { C } & 7.8278171 & -1.4308038 & 0.7670234\end{array}$
C $\quad 7.1113517 \quad-2.6076679 \quad 0.3053113$
$\begin{array}{llll}\text { C } & 8.3164023 & 0.7003328 & -0.4010951\end{array}$
$\begin{array}{llll}\text { C } & 7.8928904 & 1.4327045 & -1.5925791\end{array}$
$\begin{array}{lllll}\text { C } & 7.1488022 & 2.6127104 & -1.1558634\end{array}$
C $\quad 7.1113517 \quad 2.6076679 \quad 0.3053113$
$\begin{array}{llll}\text { C } & 7.8278171 & 1.4308038 & 0.7670234\end{array}$
$\begin{array}{lllll}\text { C } & 6.0303739 & 3.0423854 & -1.8823763\end{array}$
$\begin{array}{llll}\text { C } & 5.6103999 & 2.3155127 & -3.0751784\end{array}$
$\begin{array}{llll}\text { C } & 4.1469933 & 2.3161629 & -3.1141089\end{array}$
C $3.6656155 \quad 3.0443094-1.9443776$
$\begin{array}{llll}\text { C } & 4.8289158 & 3.4912492 & -1.1784376\end{array}$
C $\quad 3.4582546 \quad 1.1822011 \quad-3.5694123$
C $\quad 4.2018034 \quad 0.0000000-4.0025320$
C $3.4582546 \quad-1.1822011 \quad-3.5694123$
$\begin{array}{llll}\text { C } & 2.2556095 & -0.7311439 & -2.8754324\end{array}$
$\begin{array}{llll}\text { C } & 2.2556095 & 0.7311439 & -2.8754324\end{array}$ 

C $\quad 4.1469933 \quad-2.3161629 \quad-3.1141089$
$\begin{array}{llll}\text { C } & 5.6103999 & -2.3155127 & -3.0751784\end{array}$
C $\quad 6.0303739 \quad-3.0423854 \quad-1.8823763$
C $4.8289158 \quad-3.4912492 \quad-1.1784376$
C $3.6656155 \quad-3.0443094 \quad-1.9443776$
C $\quad 6.3221469-1.1819287 \quad-3.4929780$
$\begin{array}{llll}\text { C } & 5.6028490 & 0.0000000 & -3.9652691\end{array}$
C $\quad 6.3221469 \quad 1.1819287 \quad-3.4929780$
$\begin{array}{llll}\text { C } & 7.4872806 & 0.7317249 & -2.7366513\end{array}$
$\begin{array}{llll}\text { C } & 7.4872806 & -0.7317249 & -2.7366513\end{array}$
C $\quad 4.6808533 \quad 0.0000000 \quad 4.5264119$
C $\quad 4.6622955 \quad-1.2245312 \quad 5.2870086$
C $\quad 4.6622955 \quad 1.2245312 \quad 5.2870086$
N $\quad 4.6492617 \quad-2.2436062 \quad 5.8705262$
$\mathrm{N} \quad 4.6492617 \quad 2.2436062 \quad 5.8705262$

g. $4 \mathrm{C}_{2 \mathrm{~h}}$

130

$\begin{array}{llll}\text { C } & 5.6555509 & 1.8906898 & 0.7299321\end{array}$

C $\quad 4.2835086 \quad 1.8938943 \quad 1.1968535$

C $\quad 3.3955342 \quad 2.0249345 \quad 0.0000000$

C $\quad 4.2835086 \quad 1.8938943-1.1968535$

$\begin{array}{llll}\text { C } & 5.6555509 & 1.8906898 & -0.7299321\end{array}$

$\begin{array}{llll}\text { C } & 1.3078607 & -0.9717771 & 0.7298508\end{array}$

$\begin{array}{llll}\text { C } & 1.3078607 & -0.9717771 & -0.7298508\end{array}$

C $\quad 1.8449822 \quad 0.2906085-1.1958863$

$\begin{array}{llll}\text { C } & 2.0738874 & 1.1597781 & 0.0000000\end{array}$

C $\quad 1.8449822 \quad 0.2906085 \quad 1.1958863$

$\begin{array}{llll}\text { C } & 2.9657990 & -0.9138443 & -3.0302937\end{array}$ 

$\begin{array}{llll}\text { C } & 4.3549315 & -0.8746382 & -3.4795681\end{array}$
$\begin{array}{llll}\text { C } & 4.9423060 & 0.3844682 & -3.0296495\end{array}$
C $\quad 3.9232206 \quad 1.1317745 \quad-2.3035011$
$\begin{array}{llll}\text { C } & 2.6846113 & 0.3189674 & -2.3038116\end{array}$
$\begin{array}{llll}\text { C } & 7.0840440 & -0.8036375 & -2.6128328\end{array}$
C $7.9475869-0.7826217 \quad-1.4332490$
$\begin{array}{llll}\text { C } & 7.6777722 & 0.4523194 & -0.7004399\end{array}$
$\begin{array}{llll}\text { C } & 6.6476419 & 1.1878678 & -1.4309338\end{array}$
$\begin{array}{llll}\text { C } & 6.2825641 & 0.4180044 & -2.6083559\end{array}$
$\begin{array}{llll}\text { C } & 7.9475869 & -0.7826217 & 1.4332490\end{array}$
$\begin{array}{llll}\text { C } & 7.0840440 & -0.8036375 & 2.6128328\end{array}$
$\begin{array}{llll}\text { C } & 6.2825641 & 0.4180044 & 2.6083559\end{array}$
$\begin{array}{llll}\text { C } & 6.6476419 & 1.1878678 & 1.4309338\end{array}$
$\begin{array}{llll}\text { C } & 7.6777722 & 0.4523194 & 0.7004399\end{array}$
$\begin{array}{llll}\text { C } & 4.3549315 & -0.8746382 & 3.4795681\end{array}$
$\begin{array}{llll}\text { C } & 2.9657990 & -0.9138443 & 3.0302937\end{array}$
C $\quad 2.6846113 \quad 0.3189674 \quad 2.3038116$
C $\quad 3.9232206 \quad 1.1317745 \quad 2.3035011$
C $\quad 4.9423060 \quad 0.3844682 \quad 3.0296495$
$\begin{array}{llll}\text { C } & 3.2060596 & -3.3526299 & 2.6144374\end{array}$
C $\quad 2.8447064 \quad-4.1363185 \quad 1.4335422$
C $\quad 1.8219547 \quad-3.3973991 \quad 0.6997695$
C $\quad \begin{array}{lll}1.5568502 & -2.1595319 & 1.4301131\end{array}$
$\begin{array}{llll}\text { C } & 2.4046570 & -2.1308537 & 2.6083647\end{array}$
C $\quad 1.8219547 \quad-3.3973991 \quad-0.6997695$
C $\quad 2.8447064 \quad-4.1363185 \quad-1.4335422$
$\begin{array}{llll}\text { C } & 3.2060596 & -3.3526299 & -2.6144374\end{array}$
$\begin{array}{llll}\text { C } & 2.4046570 & -2.1308537 & -2.6083647\end{array}$
C $\quad 1.5568502 \quad-2.1595319 \quad-1.4301131$
$\begin{array}{llll}\text { C } & 4.5397047 & -3.3107083 & -3.0441082\end{array}$
$\begin{array}{llll}\text { C } & 5.5654610 & -4.0501197 & -2.3160981\end{array}$ 

C $\quad 6.7878792 \quad-3.2463362 \quad-2.3156679$
$\begin{array}{llll}\text { C } & 6.5163454 & -2.0110075 & -3.0429947\end{array}$
$\begin{array}{llll}\text { C } & 5.1240599 & -2.0462730 & -3.4890928\end{array}$
C $\quad 7.6134559 \quad-3.2260339-1.1820325$
$\begin{array}{llll}\text { C } & 7.2500415 & -4.0049668 & 0.0000000\end{array}$
$\begin{array}{llll}\text { C } & 7.6134559 & -3.2260339 & 1.1820325\end{array}$
$\begin{array}{llll}\text { C } & 8.2048119 & -1.9689699 & 0.7313433\end{array}$
$\begin{array}{llll}\text { C } & 8.2048119 & -1.9689699 & -0.7313433\end{array}$
$\begin{array}{llll}\text { C } & 6.7878792 & -3.2463362 & 2.3156679\end{array}$
C $\quad 5.5654610 \quad-4.0501197 \quad 2.3160981$
$\begin{array}{llll}\text { C } & 4.5397047 & -3.3107083 & 3.0441082\end{array}$
$\begin{array}{llll}\text { C } & 5.1240599 & -2.0462730 & 3.4890928\end{array}$
$\begin{array}{llll}\text { C } & 6.5163454 & -2.0110075 & 3.0429947\end{array}$
C $\quad 5.2196566 \quad-4.8001829 \quad 1.1824542$
C $\quad 6.0778646 \quad-4.7738770 \quad 0.0000000$
$\begin{array}{llll}\text { C } & 5.2196566 & -4.8001829 & -1.1824542\end{array}$
$\begin{array}{llll}\text { C } & 3.8310547 & -4.8437186 & -0.7313344\end{array}$
C $\quad 3.8310547 \quad-4.8437186 \quad 0.7313344$
$\begin{array}{llll}\text { C } & 1.9960364 & 2.7231581 & 0.0000000\end{array}$
C $\quad 1.5854853 \quad 3.3633872 \quad 1.2237485$
C $\quad \begin{array}{llll}1.5854853 & 3.3633872 & -1.2237485\end{array}$
$\begin{array}{llll}\mathrm{N} & 1.2714785 & 3.8519069 & 2.2445473\end{array}$
$\mathrm{N} \quad 1.2714785 \quad 3.8519069 \quad-2.2445473$

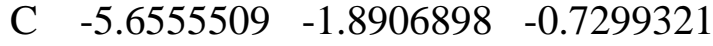
C $\quad-4.2835086-1.8938943-1.1968535$
C $\quad-3.3955342 \quad-2.0249345 \quad 0.0000000$
$\begin{array}{llll}\text { C } & -4.2835086 & -1.8938943 & 1.1968535\end{array}$
$\begin{array}{llll}\text { C } & -5.6555509 & -1.8906898 & 0.7299321\end{array}$
$\begin{array}{llll}\text { C } & -1.3078607 & 0.9717771 & -0.7298508\end{array}$
$\begin{array}{llll}\text { C } & -1.3078607 & 0.9717771 & 0.7298508\end{array}$
$\begin{array}{llll}\text { C } & -1.8449822 & -0.2906085 & 1.1958863\end{array}$ 

C $\quad-2.0738874-1.1597781 \quad 0.0000000$
C $\quad-1.8449822-0.2906085-1.1958863$
$\begin{array}{llll}\text { C } & -2.9657990 & 0.9138443 & 3.0302937\end{array}$
$\begin{array}{llll}\text { C } & -4.3549315 & 0.8746382 & 3.4795681\end{array}$
$\begin{array}{llll}\text { C } & -4.9423060 & -0.3844682 & 3.0296495\end{array}$
C $\quad-3.9232206-1.1317745 \quad 2.3035011$
C $\quad-2.6846113 \quad-0.3189674 \quad 2.3038116$
$\begin{array}{llll}\text { C } & -7.0840440 & 0.8036375 & 2.6128328\end{array}$
$\begin{array}{llll}\text { C } & -7.9475869 & 0.7826217 & 1.4332490\end{array}$
C $\quad-7.6777722 \quad-0.4523194 \quad 0.7004399$
$\begin{array}{llll}\text { C } & -6.6476419 & -1.1878678 & 1.4309338\end{array}$
C $\quad-6.2825641 \quad-0.4180044 \quad 2.6083559$
$\begin{array}{llll}\text { C } & -7.9475869 & 0.7826217 & -1.4332490\end{array}$
$\begin{array}{llll}\text { C } & -7.0840440 & 0.8036375 & -2.6128328\end{array}$
C $\quad-6.2825641 \quad-0.4180044 \quad-2.6083559$
C $\quad-6.6476419-1.1878678-1.4309338$
C $\quad-7.6777722 \quad-0.4523194 \quad-0.7004399$
$\begin{array}{llll}\text { C } & -4.3549315 & 0.8746382 & -3.4795681\end{array}$
$\begin{array}{llll}\text { C } & -2.9657990 & 0.9138443 & -3.0302937\end{array}$
C $\quad-2.6846113-0.3189674 \quad-2.3038116$
C $\quad-3.9232206-1.1317745 \quad-2.3035011$
$\begin{array}{llll}\text { C } & -4.9423060 & -0.3844682 & -3.0296495\end{array}$
$\begin{array}{llll}\text { C } & -3.2060596 & 3.3526299 & -2.6144374\end{array}$
C $\quad-2.8447064 \quad 4.1363185 \quad-1.4335422$
$\begin{array}{lllll}\text { C } & -1.8219547 & 3.3973991 & -0.6997695\end{array}$

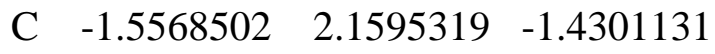
$\begin{array}{llll}\text { C } & -2.4046570 & 2.1308537 & -2.6083647\end{array}$
$\begin{array}{llll}\text { C } & -1.8219547 & 3.3973991 & 0.6997695\end{array}$
$\begin{array}{llll}\text { C } & -2.8447064 & 4.1363185 & 1.4335422\end{array}$
$\begin{array}{llll}\text { C } & -3.2060596 & 3.3526299 & 2.6144374\end{array}$
$\begin{array}{llll}\text { C } & -2.4046570 & 2.1308537 & 2.6083647\end{array}$ 


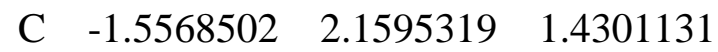
$\begin{array}{llll}\text { C } & -4.5397047 & 3.3107083 & 3.0441082\end{array}$
$\begin{array}{llll}\text { C } & -5.5654610 & 4.0501197 & 2.3160981\end{array}$
C $\quad-6.7878792 \quad 3.2463362 \quad 2.3156679$
C $\quad-6.5163454 \quad 2.0110075 \quad 3.0429947$
$\begin{array}{llll}\text { C } & -5.1240599 & 2.0462730 & 3.4890928\end{array}$

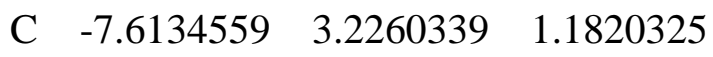
$\begin{array}{llll}\text { C } & -7.2500415 & 4.0049668 & 0.0000000\end{array}$
C $\quad \begin{array}{llll}-7.6134559 & 3.2260339 & -1.1820325\end{array}$
$\begin{array}{llll}\text { C } & -8.2048119 & 1.9689699 & -0.7313433\end{array}$
$\begin{array}{llll}\text { C } & -8.2048119 & 1.9689699 & 0.7313433\end{array}$
$\begin{array}{llll}\text { C } & -6.7878792 & 3.2463362 & -2.3156679\end{array}$
$\begin{array}{llll}\text { C } & -5.5654610 & 4.0501197 & -2.3160981\end{array}$
$\begin{array}{llll}\text { C } & -4.5397047 & 3.3107083 & -3.0441082\end{array}$
$\begin{array}{llll}\text { C } & -5.1240599 & 2.0462730 & -3.4890928\end{array}$
$\begin{array}{llll}\text { C } & -6.5163454 & 2.0110075 & -3.0429947\end{array}$
$\begin{array}{llll}\text { C } & -5.2196566 & 4.8001829 & -1.1824542\end{array}$
$\begin{array}{llll}\text { C } & -6.0778646 & 4.7738770 & 0.0000000\end{array}$
$\begin{array}{llll}\text { C } & -5.2196566 & 4.8001829 & 1.1824542\end{array}$
$\begin{array}{llll}\text { C } & -3.8310547 & 4.8437186 & 0.7313344\end{array}$
$\begin{array}{llll}\text { C } & -3.8310547 & 4.8437186 & -0.7313344\end{array}$
$\begin{array}{llll}\text { C } & -1.9960364 & -2.7231581 & 0.0000000\end{array}$
C $\quad-1.5854853-3.3633872 \quad-1.2237485$
C $\quad-1.5854853 \quad-3.3633872 \quad 1.2237485$
$\begin{array}{llll}\mathrm{N} & -1.2714785 & -3.8519069 & -2.2445473\end{array}$
$\begin{array}{llll}\mathrm{N} & -1.2714785 & -3.8519069 & 2.2445473\end{array}$

h. $5 \mathrm{C}_{2 \mathrm{v}}$

130

C $\quad 5.5567581 \quad 2.6061772 \quad 1.9551270$

C $\quad 5.5639702 \quad 1.4602334 \quad 2.8426339$ 

$\begin{array}{llll}\text { C } & 6.8954685 & 0.7895919 & 2.7141935\end{array}$
C $\quad 7.5893179 \quad 1.4593160 \quad 1.5701096$
$\begin{array}{llll}\text { C } & 6.7934864 & 2.6060812 & 1.1785748\end{array}$
C $\quad 5.5567581 \quad-2.6061772 \quad 1.9551270$
$\begin{array}{llll}\text { C } & 6.7934864 & -2.6060812 & 1.1785748\end{array}$
C $7.5893179-1.4593160 \quad 1.5701096$
$\begin{array}{llll}\text { C } & 6.8954685 & -0.7895919 & 2.7141935\end{array}$
C $\quad 5.5639702 \quad-1.4602334 \quad 2.8426339$
$\begin{array}{llll}\text { C } & 8.2780712 & -1.1825745 & -0.7796809\end{array}$
C $\quad 8.2699283 \quad 0.0000000 \quad-1.6363771$
$\begin{array}{llll}\text { C } & 8.2780712 & 1.1825745 & -0.7796809\end{array}$
$\begin{array}{llll}\text { C } & 8.2926920 & 0.7407951 & 0.6090799\end{array}$
$\begin{array}{llll}\text { C } & 8.2926920 & -0.7407951 & 0.6090799\end{array}$
$\begin{array}{llll}\text { C } & 6.7723649 & 2.3204634 & -2.3948155\end{array}$
$\begin{array}{lllll}\text { C } & 5.5303013 & 3.0529924 & -2.1533871\end{array}$
$\begin{array}{llll}\text { C } & 5.5378765 & 3.5054522 & -0.7645987\end{array}$
$\begin{array}{llll}\text { C } & 6.7843368 & 3.0485689 & -0.1535123\end{array}$
C $\quad 7.5448174 \quad 2.3205503 \quad-1.1547902$
$\begin{array}{llll}\text { C } & 3.1036410 & 3.0537027 & -0.6290282\end{array}$
$\begin{array}{llll}\text { C } & 2.3473283 & 2.3211287 & 0.3857725\end{array}$
$\begin{array}{llll}\text { C } & 3.1288240 & 2.3212750 & 1.6201438\end{array}$
C $\quad 4.3608033 \quad 3.0494418 \quad 1.3692786$
$\begin{array}{lllll}\text { C } & 4.3513192 & 3.5058381 & -0.0194722\end{array}$
C $\quad 2.3796657 \quad 0.0000000 \quad 2.0651922$
C $\quad 3.1484776 \quad-1.1829801 \quad 2.4438935$
$\begin{array}{llll}\text { C } & 4.3934591 & -0.7410110 & 3.0592248\end{array}$
$\begin{array}{llll}\text { C } & 4.3934591 & 0.7410110 & 3.0592248\end{array}$
C $\quad 3.1484776 \quad 1.1829801 \quad 2.4438935$
C $\quad 2.3473283 \quad-2.3211287 \quad 0.3857725$
$\begin{array}{llll}\text { C } & 3.1036410 & -3.0537027 & -0.6290282\end{array}$
$\begin{array}{llll}\text { C } & 4.3513192 & -3.5058381 & -0.0194722\end{array}$ 

C $\quad 4.3608033 \quad-3.0494418 \quad 1.3692786$
C $\quad 3.1288240 \quad-2.3212750 \quad 1.6201438$
$\begin{array}{llll}\text { C } & 5.5378765 & -3.5054522 & -0.7645987\end{array}$
C $\quad 5.5303013 \quad-3.0529924 \quad-2.1533871$
$\begin{array}{llll}\text { C } & 6.7723649 & -2.3204634 & -2.3948155\end{array}$
C $\quad 7.5448174 \quad-2.3205503 \quad-1.1547902$
$\begin{array}{llll}\text { C } & 6.7843368 & -3.0485689 & -0.1535123\end{array}$
C $\quad 6.7649494 \quad-1.1829256 \quad-3.2138652$
$\begin{array}{llll}\text { C } & 5.5209040 & -0.7316927 & -3.8281209\end{array}$
$\begin{array}{llll}\text { C } & 5.5209040 & 0.7316927 & -3.8281209\end{array}$
C $\quad 6.7649494 \quad 1.1829256 \quad-3.2138652$
$\begin{array}{llll}\text { C } & 7.5339446 & 0.0000000 & -2.8287131\end{array}$
$\begin{array}{llll}\text { C } & 4.3286414 & 1.4328955 & -3.5955238\end{array}$
$\begin{array}{llll}\text { C } & 3.0876199 & 0.7010302 & -3.3489441\end{array}$
$\begin{array}{lllll}\text { C } & 2.3270447 & 1.4330944 & -2.3379634\end{array}$
C $3.0947391 \quad 2.6178235 \quad-1.9623535$
$\begin{array}{llll}\text { C } & 4.3333992 & 2.6169662 & -2.7400358\end{array}$
C $\quad 1.6048316 \quad 0.7310409 \quad-1.3632944$
$\begin{array}{llll}\text { C } & 1.6048316 & -0.7310409 & -1.3632944\end{array}$
C $\quad 1.6165105 \quad-1.1825950 \quad 0.0234793$
$\begin{array}{llll}\text { C } & 1.6298761 & 0.0000000 & 0.8823510\end{array}$
C $\quad 1.6165105 \quad 1.1825950 \quad 0.0234793$
$\begin{array}{lllll}\text { C } & 2.3270447 & -1.4330944 & -2.3379634\end{array}$
C $\quad 3.0876199 \quad-0.7010302 \quad-3.3489441$
C $\quad 4.3286414 \quad-1.4328955 \quad-3.5955238$
$\begin{array}{llll}\text { C } & 4.3333992 & -2.6169662 & -2.7400358\end{array}$
$\begin{array}{llll}\text { C } & 3.0947391 & -2.6178235 & -1.9623535\end{array}$
C $\quad 7.6148761 \quad 0.0000000 \quad 3.8575681$
C $\quad 6.9812448 \quad 0.0000000 \quad 5.1523837$
C $\quad 9.0562565 \quad 0.0000000 \quad 3.8514758$
$\begin{array}{llll}\mathrm{N} & 6.4261181 & 0.0000000 & 6.1873368\end{array}$ 

$\begin{array}{llll}\mathrm{N} & 10.2297184 & 0.0000000 & 3.8045330\end{array}$
$\begin{array}{llll}\text { C } & -6.7934864 & 2.6060812 & 1.1785748\end{array}$

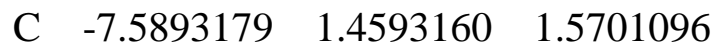
$\begin{array}{llll}\text { C } & -6.8954685 & 0.7895919 & 2.7141935\end{array}$
$\begin{array}{llll}\text { C } & -5.5639702 & 1.4602334 & 2.8426339\end{array}$
$\begin{array}{llll}\text { C } & -5.5567581 & 2.6061772 & 1.9551270\end{array}$
$\begin{array}{llll}\text { C } & -6.7934864 & -2.6060812 & 1.1785748\end{array}$
C $\quad-5.5567581 \quad-2.6061772 \quad 1.9551270$
$\begin{array}{llll}\text { C } & -5.5639702 & -1.4602334 & 2.8426339\end{array}$
C $\quad-6.8954685 \quad-0.7895919 \quad 2.7141935$
C $\quad-7.5893179 \quad-1.4593160 \quad 1.5701096$
C $\quad-3.1484776-1.1829801 \quad 2.4438935$
$\begin{array}{llll}\text { C } & -2.3796657 & 0.0000000 & 2.0651922\end{array}$
$\begin{array}{llll}\text { C } & -3.1484776 & 1.1829801 & 2.4438935\end{array}$
$\begin{array}{llll}\text { C } & -4.3934591 & 0.7410110 & 3.0592248\end{array}$
C $\quad-4.3934591 \quad-0.7410110 \quad 3.0592248$
$\begin{array}{llll}\text { C } & -2.3473283 & 2.3211287 & 0.3857725\end{array}$
$\begin{array}{llll}\text { C } & -3.1036410 & 3.0537027 & -0.6290282\end{array}$
$\begin{array}{llll}\text { C } & -4.3513192 & 3.5058381 & -0.0194722\end{array}$
$\begin{array}{llll}\text { C } & -4.3608033 & 3.0494418 & 1.3692786\end{array}$
$\begin{array}{llll}\text { C } & -3.1288240 & 2.3212750 & 1.6201438\end{array}$
C $\quad-5.5303013 \quad 3.0529924 \quad-2.1533871$
C $\quad-6.7723649 \quad 2.3204634 \quad-2.3948155$
C $\quad-7.5448174 \quad 2.3205503 \quad-1.1547902$
C $\quad-6.7843368 \quad 3.0485689 \quad-0.1535123$
$\begin{array}{llll}\text { C } & -5.5378765 & 3.5054522 & -0.7645987\end{array}$
$\begin{array}{llll}\text { C } & -8.2699283 & 0.0000000 & -1.6363771\end{array}$
$\begin{array}{llll}\text { C } & -8.2780712 & -1.1825745 & -0.7796809\end{array}$
$\begin{array}{llll}\text { C } & -8.2926920 & -0.7407951 & 0.6090799\end{array}$
$\begin{array}{llll}\text { C } & -8.2926920 & 0.7407951 & 0.6090799\end{array}$
$\begin{array}{llll}\text { C } & -8.2780712 & 1.1825745 & -0.7796809\end{array}$ 

C $\quad-6.7723649 \quad-2.3204634 \quad-2.3948155$
C $\quad-5.5303013 \quad-3.0529924 \quad-2.1533871$
$\begin{array}{llll}\text { C } & -5.5378765 & -3.5054522 & -0.7645987\end{array}$
C $\quad-6.7843368-3.0485689-0.1535123$
C $\quad-7.5448174 \quad-2.3205503 \quad-1.1547902$
$\begin{array}{llll}\text { C } & -4.3513192 & -3.5058381 & -0.0194722\end{array}$
$\begin{array}{llll}\text { C } & -3.1036410 & -3.0537027 & -0.6290282\end{array}$
$\begin{array}{llll}\text { C } & -2.3473283 & -2.3211287 & 0.3857725\end{array}$
C $\quad-3.1288240 \quad-2.3212750 \quad 1.6201438$
$\begin{array}{llll}\text { C } & -4.3608033 & -3.0494418 & 1.3692786\end{array}$
C $\quad-1.6165105-1.1825950 \quad 0.0234793$
$\begin{array}{llll}\text { C } & -1.6048316 & -0.7310409 & -1.3632944\end{array}$
$\begin{array}{llll}\text { C } & -1.6048316 & 0.7310409 & -1.3632944\end{array}$
$\begin{array}{llll}\text { C } & -1.6165105 & 1.1825950 & 0.0234793\end{array}$
$\begin{array}{llll}\text { C } & -1.6298761 & 0.0000000 & 0.8823510\end{array}$
$\begin{array}{lllll}\text { C } & -2.3270447 & 1.4330944 & -2.3379634\end{array}$
$\begin{array}{llll}\text { C } & -3.0876199 & 0.7010302 & -3.3489441\end{array}$
C $\quad-4.3286414 \quad 1.4328955 \quad-3.5955238$
$\begin{array}{llll}\text { C } & -4.3333992 & 2.6169662 & -2.7400358\end{array}$
$\begin{array}{llll}\text { C } & -3.0947391 & 2.6178235 & -1.9623535\end{array}$
$\begin{array}{llll}\text { C } & -5.5209040 & 0.7316927 & -3.8281209\end{array}$
$\begin{array}{llll}\text { C } & -5.5209040 & -0.7316927 & -3.8281209\end{array}$
C $\quad-6.7649494-1.1829256-3.2138652$
$\begin{array}{llll}\text { C } & -7.5339446 & 0.0000000 & -2.8287131\end{array}$
C $\quad-6.7649494 \quad 1.1829256 \quad-3.2138652$
C $\quad-4.3286414-1.4328955-3.5955238$
C $\quad-3.0876199-0.7010302 \quad-3.3489441$
$\begin{array}{llll}\text { C } & -2.3270447 & -1.4330944 & -2.3379634\end{array}$
C $\quad-3.0947391 \quad-2.6178235 \quad-1.9623535$
$\begin{array}{llll}\text { C } & -4.3333992 & -2.6169662 & -2.7400358\end{array}$
$\begin{array}{llll}\text { C } & -7.6148761 & 0.0000000 & 3.8575681\end{array}$ 

$\begin{array}{llll}\text { C } & -9.0562565 & 0.0000000 & 3.8514758\end{array}$
$\begin{array}{llll}\text { C } & -6.9812448 & 0.0000000 & 5.1523837\end{array}$
$\begin{array}{llll}\mathrm{N} & -10.2297184 & 0.0000000 & 3.8045330\end{array}$
$\begin{array}{llll}\mathrm{N} & -6.4261181 & 0.0000000 & 6.1873368\end{array}$

\section{i. $6 \mathrm{C}_{2 \mathrm{v}}$}

130
C $\quad-5.5547329-2.6061832 \quad 1.9554466$
$\begin{array}{llll}\text { C } & -5.5611794 & -1.4602347 & 2.8429572\end{array}$
$\begin{array}{llll}\text { C } & -6.8927950 & -0.7895913 & 2.7156509\end{array}$
$\begin{array}{llll}\text { C } & -7.5876209 & -1.4593118 & 1.5721714\end{array}$
C $\quad-6.7921197 \quad-2.6060745 \quad 1.1799461$
$\begin{array}{llll}\text { C } & -5.5547329 & 2.6061832 & 1.9554466\end{array}$
$\begin{array}{llll}\text { C } & -6.7921197 & 2.6060745 & 1.1799461\end{array}$
$\begin{array}{llll}\text { C } & -7.5876209 & 1.4593118 & 1.5721714\end{array}$
$\begin{array}{llll}\text { C } & -6.8927950 & 0.7895913 & 2.7156509\end{array}$
$\begin{array}{llll}\text { C } & -5.5611794 & 1.4602347 & 2.8429572\end{array}$
C $\quad-8.2783876 \quad 1.1825723 \quad-0.7770324$
$\begin{array}{lrrrr}\text { C } & -8.2709918 & 0.0000000 & -1.6337395\end{array}$
C $\quad-8.2783876-1.1825723-0.7770324$
$\begin{array}{lrrr}\text { C } & -8.2918146 & -0.7407980 & 0.6117390\end{array}$
$\begin{array}{llll}\text { C } & -8.2918146 & 0.7407980 & 0.6117390\end{array}$
$\begin{array}{llll}\text { C } & -6.7740709 & -2.3204664 & -2.3934600\end{array}$
$\begin{array}{lrrr}\text { C } & -5.5317950 & -3.0529900 & -2.1530961\end{array}$
$\begin{array}{llll}\text { C } & -5.5381800 & -3.5054473 & -0.7643027\end{array}$
C $\quad-6.7841191 \quad-3.0485656 \quad-0.1521441$
$\begin{array}{llll}\text { C } & -7.5454579 & -2.3205510 & -1.1527682\end{array}$
$\begin{array}{llll}\text { C } & -3.1038286 & -3.0536990 & -0.6308222\end{array}$
$\begin{array}{llll}\text { C } & -2.3466525 & -2.3211283 & 0.3833284\end{array}$ 

C $\quad-3.1270863 \quad-2.3212795 \quad 1.6183771$
$\begin{array}{llll}\text { C } & -4.3592815 & -3.0494362 & 1.3685615\end{array}$
C $\quad-4.3509881 \quad-3.5058329 \quad-0.0201939$
$\begin{array}{llll}\text { C } & -2.3775616 & 0.0000000 & 2.0627744\end{array}$
C $\quad-3.1460343 \quad 1.1829831 \quad 2.4421451$
$\begin{array}{llll}\text { C } & -4.3904856 & 0.7410124 & 3.0585496\end{array}$
C $\quad-4.3904856-0.7410124 \quad 3.0585496$
$\begin{array}{llll}\text { C } & -3.1460343 & -1.1829831 & 2.4421451\end{array}$
$\begin{array}{llll}\text { C } & -2.3466525 & 2.3211283 & 0.3833284\end{array}$
$\begin{array}{llll}\text { C } & -3.1038286 & 3.0536990 & -0.6308222\end{array}$
$\begin{array}{llll}\text { C } & -4.3509881 & 3.5058329 & -0.0201939\end{array}$
$\begin{array}{llll}\text { C } & -4.3592815 & 3.0494362 & 1.3685615\end{array}$
C $\quad-3.1270863 \quad 2.3212795 \quad 1.6183771$
$\begin{array}{llll}\text { C } & -5.5381800 & 3.5054473 & -0.7643027\end{array}$
$\begin{array}{llll}\text { C } & -5.5317950 & 3.0529900 & -2.1530961\end{array}$
$\begin{array}{llll}\text { C } & -6.7740709 & 2.3204664 & -2.3934600\end{array}$
C $\quad-7.5454579 \quad 2.3205510 \quad-1.1527682$
C $\quad-6.7841191 \quad 3.0485656 \quad-0.1521441$
C $\quad-6.7673624 \quad 1.1829277 \quad-3.2125191$
$\begin{array}{llll}\text { C } & -5.5238373 & 0.7316899 & -3.8278324\end{array}$
C $\quad-5.5238373 \quad-0.7316899 \quad-3.8278324$
C $\quad-6.7673624 \quad-1.1829277 \quad-3.2125191$
$\begin{array}{llll}\text { C } & -7.5360258 & 0.0000000 & -2.8267111\end{array}$
C $\quad-4.3313733 \quad-1.4328871 \quad-3.5962591$
C $\quad-3.0901415 \quad-0.7010304 \quad-3.3507515$
C $\quad-2.3286817 \quad-1.4330969-2.3404311$
C $\quad-3.0960758 \quad-2.6178163 \quad-1.9641504$
C $\quad-4.3353963-2.6169729 \quad-2.7407745$
$\begin{array}{llll}\text { C } & -1.6056208 & -0.7310448 & -1.3663840\end{array}$
$\begin{array}{llll}\text { C } & -1.6056208 & 0.7310448 & -1.3663840\end{array}$
$\begin{array}{llll}\text { C } & -1.6161591 & 1.1825913 & 0.0204002\end{array}$ 

$\begin{array}{llll}\text { C } & -1.6288055 & 0.0000000 & 0.8792826\end{array}$
C $\quad-1.6161591 \quad-1.1825913 \quad 0.0204002$
$\begin{array}{llll}\text { C } & -2.3286817 & 1.4330969 & -2.3404311\end{array}$
$\begin{array}{llll}\text { C } & -3.0901415 & 0.7010304 & -3.3507515\end{array}$
C $\quad-4.3313733 \quad 1.4328871 \quad-3.5962591$
C $\quad-4.3353963 \quad 2.6169729 \quad-2.7407745$
$\begin{array}{llll}\text { C } & -3.0960758 & 2.6178163 & -1.9641504\end{array}$
C $\quad-7.6112133 \quad 0.0000000 \quad 3.8596496$
$\begin{array}{llll}\text { C } & -6.9764361 & 0.0000000 & 5.1539017\end{array}$
$\begin{array}{llll}\text { C } & -9.0525981 & 0.0000000 & 3.8548248\end{array}$
$\mathrm{N} \quad-6.4203762 \quad 0.0000000 \quad 6.1883536$
$\begin{array}{llll}\mathrm{N} & -10.2261029 & 0.0000000 & 3.8089607\end{array}$
$\begin{array}{llll}\text { C } & 5.5547329 & -2.6061832 & 1.9554466\end{array}$
$\begin{array}{llll}\text { C } & 5.5611794 & -1.4602347 & 2.8429572\end{array}$
C $\quad \begin{array}{llll}6.8927950 & -0.7895913 & 2.7156509\end{array}$
$\begin{array}{llll}\text { C } & 7.5876209 & -1.4593118 & 1.5721714\end{array}$
$\begin{array}{llll}\text { C } & 6.7921197 & -2.6060745 & 1.1799461\end{array}$
C $\quad 5.5547329 \quad 2.6061832 \quad 1.9554466$
$\begin{array}{llll}\text { C } & 6.7921197 & 2.6060745 & 1.1799461\end{array}$
$\begin{array}{llll}\text { C } & 7.5876209 & 1.4593118 & 1.5721714\end{array}$
$\begin{array}{llll}\text { C } & 6.8927950 & 0.7895913 & 2.7156509\end{array}$
$\begin{array}{llll}\text { C } & 5.5611794 & 1.4602347 & 2.8429572\end{array}$
C $\quad 8.2783876 \quad 1.1825723 \quad-0.7770324$
$\begin{array}{llll}\text { C } & 8.2709918 & 0.0000000 & -1.6337395\end{array}$
C $\quad 8.2783876 \quad-1.1825723 \quad-0.7770324$
$\begin{array}{llll}\text { C } & 8.2918146 & -0.7407980 & 0.6117390\end{array}$
$\begin{array}{llll}\text { C } & 8.2918146 & 0.7407980 & 0.6117390\end{array}$
$\begin{array}{llll}\text { C } & 6.7740709 & -2.3204664 & -2.3934600\end{array}$
C $\quad 5.5317950 \quad-3.0529900 \quad-2.1530961$
$\begin{array}{llll}\text { C } & 5.5381800 & -3.5054473 & -0.7643027\end{array}$
$\begin{array}{llll}\text { C } & 6.7841191 & -3.0485656 & -0.1521441\end{array}$ 

C $\quad 7.5454579 \quad-2.3205510 \quad-1.1527682$
$\begin{array}{llll}\text { C } & 3.1038286 & -3.0536990 & -0.6308222\end{array}$
$\begin{array}{llll}\text { C } & 2.3466525 & -2.3211283 & 0.3833284\end{array}$
$\begin{array}{llll}\text { C } & 3.1270863 & -2.3212795 & 1.6183771\end{array}$
$\begin{array}{llll}\text { C } & 4.3592815 & -3.0494362 & 1.3685615\end{array}$
$\begin{array}{llll}\text { C } & 4.3509881 & -3.5058329 & -0.0201939\end{array}$
$\begin{array}{llll}\text { C } & 2.3775616 & 0.0000000 & 2.0627744\end{array}$
$\begin{array}{llll}\text { C } & 3.1460343 & 1.1829831 & 2.4421451\end{array}$
C $\quad 4.3904856 \quad 0.7410124 \quad 3.0585496$
C $\quad 4.3904856-0.7410124 \quad 3.0585496$
C $\quad 3.1460343 \quad-1.1829831 \quad 2.4421451$
$\begin{array}{llll}\text { C } & 2.3466525 & 2.3211283 & 0.3833284\end{array}$
$\begin{array}{llll}\text { C } & 3.1038286 & 3.0536990 & -0.6308222\end{array}$
$\begin{array}{llll}\text { C } & 4.3509881 & 3.5058329 & -0.0201939\end{array}$
C $\quad 4.3592815 \quad 3.0494362 \quad 1.3685615$
C $\quad 3.1270863 \quad 2.3212795 \quad 1.6183771$
C $\quad 5.5381800 \quad 3.5054473 \quad-0.7643027$
$\begin{array}{llll}\text { C } & 5.5317950 & 3.0529900 & -2.1530961\end{array}$
$\begin{array}{llll}C & 6.7740709 & 2.3204664 & -2.3934600\end{array}$
C $\quad 7.5454579 \quad 2.3205510 \quad-1.1527682$
C $\quad 6.7841191 \quad 3.0485656 \quad-0.1521441$
$\begin{array}{llll}\text { C } & 6.7673624 & 1.1829277 & -3.2125191\end{array}$
$\begin{array}{llll}\text { C } & 5.5238373 & 0.7316899 & -3.8278324\end{array}$
$\begin{array}{llll}\text { C } & 5.5238373 & -0.7316899 & -3.8278324\end{array}$
C $\quad 6.7673624 \quad-1.1829277 \quad-3.2125191$
C $\quad 7.5360258 \quad 0.0000000 \quad-2.8267111$
C $4.3313733 \quad-1.4328871 \quad-3.5962591$
$\begin{array}{llll}\text { C } & 3.0901415 & -0.7010304 & -3.3507515\end{array}$
$\begin{array}{llll}\text { C } & 2.3286817 & -1.4330969 & -2.3404311\end{array}$
C $\quad 3.0960758 \quad-2.6178163 \quad-1.9641504$
$\begin{array}{llll}\text { C } & 4.3353963 & -2.6169729 & -2.7407745\end{array}$ 

C $\quad 1.6056208 \quad-0.7310448 \quad-1.3663840$
C $\quad \begin{array}{llll}1.6056208 & 0.7310448 & -1.3663840\end{array}$
$\begin{array}{llll}\text { C } & 1.6161591 & 1.1825913 & 0.0204002\end{array}$
C $\quad 1.62880550 .0000000 \quad 0.8792826$
C $\quad \begin{array}{lll}1.6161591 & -1.1825913 & 0.0204002\end{array}$
$\begin{array}{llll}\text { C } & 2.3286817 & 1.4330969 & -2.3404311\end{array}$
$\begin{array}{llll}\text { C } & 3.0901415 & 0.7010304 & -3.3507515\end{array}$
$\begin{array}{llll}\text { C } & 4.3313733 & 1.4328871 & -3.5962591\end{array}$
$\begin{array}{llll}\text { C } & 4.3353963 & 2.6169729 & -2.7407745\end{array}$
$\begin{array}{llll}\text { C } & 3.0960758 & 2.6178163 & -1.9641504\end{array}$
C $\quad 7.6112133 \quad 0.0000000 \quad 3.8596496$
$\begin{array}{llll}\text { C } & 6.9764361 & 0.0000000 & 5.1539017\end{array}$
$\begin{array}{llll}\text { C } & 9.0525981 & 0.0000000 & 3.8548248\end{array}$
$\begin{array}{llll}\mathrm{N} & 6.4203762 & 0.0000000 & 6.1883536\end{array}$
$\begin{array}{llll}\mathrm{N} & 10.2261029 & 0.0000000 & 3.8089607\end{array}$

\section{j. $7 \mathrm{C}_{2 \mathrm{v}}$}

130
C $\quad 8.3157191 \quad 0.7297824 \quad-1.1632359$
C $\quad 8.3195831 \quad 1.1962214 \quad 0.2092066$
$\begin{array}{llll}\text { C } & 8.4544215 & 0.0000000 & 1.0980944\end{array}$
$\begin{array}{llll}\text { C } & 8.3195831 & -1.1962214 & 0.2092066\end{array}$
$\begin{array}{llll}\text { C } & 8.3157191 & -0.7297824 & -1.1632359\end{array}$
$\begin{array}{llll}\text { C } & 5.4561400 & 0.7300882 & 3.1932626\end{array}$
$\begin{array}{llll}\text { C } & 5.4561400 & -0.7300882 & 3.1932626\end{array}$
$\begin{array}{llll}\text { C } & 6.7164953 & -1.1956288 & 2.6499345\end{array}$
$\begin{array}{llll}\text { C } & 7.5860451 & 0.0000000 & 2.4210827\end{array}$
$\begin{array}{llll}\text { C } & 6.7164953 & 1.1956288 & 2.6499345\end{array}$
$\begin{array}{llll}\text { C } & 5.5125479 & -3.0289851 & 1.5275966\end{array}$ 

C $\quad 5.5512114 \quad-3.4783200 \quad 0.1384176$
$\begin{array}{llll}\text { C } & 6.8100039 & -3.0286652 & -0.4494414\end{array}$
$\begin{array}{llll}\text { C } & 7.5574037 & -2.3024998 & 0.5693508\end{array}$
$\begin{array}{llll}\text { C } & 6.7443404 & -2.3024744 & 1.8081374\end{array}$
C $\quad 5.6212276 \quad-2.6128635 \quad-2.5909661$
$\begin{array}{llll}\text { C } & 5.6417785 & -1.4331996 & -3.4544789\end{array}$
$\begin{array}{llll}\text { C } & 6.8768288 & -0.7004074 & -3.1851767\end{array}$
$\begin{array}{llll}\text { C } & 7.6128058 & -1.4308340 & -2.1552433\end{array}$
$\begin{array}{llll}\text { C } & 6.8431309 & -2.6081882 & -1.7899150\end{array}$
$\begin{array}{llll}\text { C } & 5.6417785 & 1.4331996 & -3.4544789\end{array}$
C $\quad 5.6212276 \quad 2.6128635 \quad-2.5909661$
$\begin{array}{llll}\text { C } & 6.8431309 & 2.6081882 & -1.7899150\end{array}$
$\begin{array}{llll}\text { C } & 7.6128058 & 1.4308340 & -2.1552433\end{array}$
$\begin{array}{llll}\text { C } & 6.8768288 & 0.7004074 & -3.1851767\end{array}$
C $\quad 5.5512114 \quad 3.4783200 \quad 0.1384176$
C $\quad 5.5125479 \quad 3.0289851 \quad 1.5275966$
C $\quad 6.7443404 \quad 2.3024744 \quad 1.8081374$
$\begin{array}{llll}\text { C } & 7.5574037 & 2.3024998 & 0.5693508\end{array}$
$\begin{array}{llll}\text { C } & 6.8100039 & 3.0286652 & -0.4494414\end{array}$
$\begin{array}{llll}\text { C } & 3.0745885 & 2.6142737 & 1.2890289\end{array}$
C $\quad 2.2916776 \quad 1.4333510 \quad 1.6511804$
C $\quad 3.0289290 \quad 0.7005553 \quad 2.6774763$
$\begin{array}{llll}\text { C } & 4.2666251 & 1.4315050 & 2.9435338\end{array}$
$\begin{array}{llll}\text { C } & 4.2959932 & 2.6078920 & 2.0907379\end{array}$
$\begin{array}{llll}\text { C } & 3.0289290 & -0.7005553 & 2.6774763\end{array}$
C $\quad 2.2916776 \quad-1.4333510 \quad 1.6511804$
$\begin{array}{llll}\text { C } & 3.0745885 & -2.6142737 & 1.2890289\end{array}$
$\begin{array}{llll}\text { C } & 4.2959932 & -2.6078920 & 2.0907379\end{array}$
$\begin{array}{llll}\text { C } & 4.2666251 & -1.4315050 & 2.9435338\end{array}$
C $\quad 3.1158858 \quad-3.0439256 \quad-0.0451308$
$\begin{array}{llll}\text { C } & 2.3760014 & -2.3166309 & -1.0712386\end{array}$ 

C $\quad 3.1786538 \quad-2.3164733 \quad-2.2946397$
$\begin{array}{llll}\text { C } & 4.4140257 & -3.0434069 & -2.0231748\end{array}$
$\begin{array}{llll}\text { C } & 4.3795352 & -3.4890131 & -0.6304535\end{array}$
C $3.1984931-1.1823522 \quad-3.1200966$
$\begin{array}{llll}\text { C } & 2.4193330 & 0.0000000 & -2.7568202\end{array}$
$\begin{array}{llll}\text { C } & 3.1984931 & 1.1823522 & -3.1200966\end{array}$
C $\quad 4.4552355 \quad 0.7314673 \quad-3.7117167$
C $\quad 4.4552355 \quad-0.7314673 \quad-3.7117167$
$\begin{array}{llll}\text { C } & 3.1786538 & 2.3164733 & -2.2946397\end{array}$
$\begin{array}{llll}\text { C } & 2.3760014 & 2.3166309 & -1.0712386\end{array}$
C $\quad 3.1158858 \quad 3.0439256 \quad-0.0451308$
$\begin{array}{llll}\text { C } & 4.3795352 & 3.4890131 & -0.6304535\end{array}$
$\begin{array}{llll}\text { C } & 4.4140257 & 3.0434069 & -2.0231748\end{array}$
$\begin{array}{llll}\text { C } & 1.6297500 & 1.1819134 & -0.7248551\end{array}$
$\begin{array}{llll}\text { C } & 1.6543310 & 0.0000000 & -1.5828258\end{array}$
C $\quad 1.6297500 \quad-1.1819134 \quad-0.7248551$
C $\quad 1.5891069 \quad-0.7306594 \quad 0.6632100$
C $\quad 1.5891069 \quad 0.7306594 \quad 0.6632100$
$\begin{array}{llll}\text { C } & 9.1474251 & 0.0000000 & 2.4989148\end{array}$
C $\quad 9.7839267 \quad 1.2239825 \quad 2.9183758$
C $\quad 9.7839267 \quad-1.2239825 \quad 2.9183758$
$\mathrm{N} \quad 10.2715445 \quad 2.2424527 \quad 3.2410909$
$\mathrm{N} \quad 10.2715445 \quad-2.2424527 \quad 3.2410909$
$\begin{array}{llll}\text { C } & -8.3157191 & 0.7297824 & -1.1632359\end{array}$
$\begin{array}{llll}\text { C } & -8.3195831 & 1.1962214 & 0.2092066\end{array}$
$\begin{array}{llll}\text { C } & -8.4544215 & 0.0000000 & 1.0980944\end{array}$
C $\quad-8.3195831 \quad-1.1962214 \quad 0.2092066$
C $\quad-8.3157191 \quad-0.7297824 \quad-1.1632359$
C $\quad-5.4561400 \quad 0.7300882 \quad 3.1932626$
$\begin{array}{llll}\text { C } & -5.4561400 & -0.7300882 & 3.1932626\end{array}$
$\begin{array}{llll}\text { C } & -6.7164953 & -1.1956288 & 2.6499345\end{array}$ 

$\begin{array}{llll}\text { C } & -7.5860451 & 0.0000000 & 2.4210827\end{array}$
$\begin{array}{llll}\text { C } & -6.7164953 & 1.1956288 & 2.6499345\end{array}$
$\begin{array}{llll}\text { C } & -5.5125479 & -3.0289851 & 1.5275966\end{array}$
$\begin{array}{llll}\text { C } & -5.5512114 & -3.4783200 & 0.1384176\end{array}$
$\begin{array}{llll}\text { C } & -6.8100039 & -3.0286652 & -0.4494414\end{array}$
$\begin{array}{llll}\text { C } & -7.5574037 & -2.3024998 & 0.5693508\end{array}$
$\begin{array}{llll}\text { C } & -6.7443404 & -2.3024744 & 1.8081374\end{array}$
C $\quad-5.6212276-2.6128635 \quad-2.5909661$
$\begin{array}{llll}\text { C } & -5.6417785 & -1.4331996 & -3.4544789\end{array}$
$\begin{array}{llll}\text { C } & -6.8768288 & -0.7004074 & -3.1851767\end{array}$
$\begin{array}{llll}\text { C } & -7.6128058 & -1.4308340 & -2.1552433\end{array}$
$\begin{array}{llll}\text { C } & -6.8431309 & -2.6081882 & -1.7899150\end{array}$
$\begin{array}{llll}\text { C } & -5.6417785 & 1.4331996 & -3.4544789\end{array}$
$\begin{array}{llll}\text { C } & -5.6212276 & 2.6128635 & -2.5909661\end{array}$
$\begin{array}{llll}\text { C } & -6.8431309 & 2.6081882 & -1.7899150\end{array}$
$\begin{array}{lllll}\text { C } & -7.6128058 & 1.4308340 & -2.1552433\end{array}$
$\begin{array}{llll}\text { C } & -6.8768288 & 0.7004074 & -3.1851767\end{array}$
$\begin{array}{llll}\text { C } & -5.5512114 & 3.4783200 & 0.1384176\end{array}$
$\begin{array}{llll}\text { C } & -5.5125479 & 3.0289851 & 1.5275966\end{array}$
$\begin{array}{llll}\text { C } & -6.7443404 & 2.3024744 & 1.8081374\end{array}$
$\begin{array}{llll}\text { C } & -7.5574037 & 2.3024998 & 0.5693508\end{array}$
$\begin{array}{llll}\text { C } & -6.8100039 & 3.0286652 & -0.4494414\end{array}$
$\begin{array}{llll}\text { C } & -3.0745885 & 2.6142737 & 1.2890289\end{array}$
C $\quad-2.2916776 \quad 1.4333510 \quad 1.6511804$
$\begin{array}{llll}\text { C } & -3.0289290 & 0.7005553 & 2.6774763\end{array}$
$\begin{array}{llll}\text { C } & -4.2666251 & 1.4315050 & 2.9435338\end{array}$
$\begin{array}{llll}\text { C } & -4.2959932 & 2.6078920 & 2.0907379\end{array}$
$\begin{array}{llll}\text { C } & -3.0289290 & -0.7005553 & 2.6774763\end{array}$
C $\quad-2.2916776-1.4333510 \quad 1.6511804$
$\begin{array}{llll}\text { C } & -3.0745885 & -2.6142737 & 1.2890289\end{array}$
$\begin{array}{llll}\text { C } & -4.2959932 & -2.6078920 & 2.0907379\end{array}$ 

C $\quad-4.2666251 \quad-1.4315050 \quad 2.9435338$
$\begin{array}{llll}\text { C } & -3.1158858 & -3.0439256 & -0.0451308\end{array}$
$\begin{array}{llll}\text { C } & -2.3760014 & -2.3166309 & -1.0712386\end{array}$
C $\quad-3.1786538-2.3164733 \quad-2.2946397$
$\begin{array}{llll}\text { C } & -4.4140257 & -3.0434069 & -2.0231748\end{array}$
C $\quad-4.3795352-3.4890131 \quad-0.6304535$
C $\quad-3.1984931 \quad-1.1823522 \quad-3.1200966$
$\begin{array}{llll}\text { C } & -2.4193330 & 0.0000000 & -2.7568202\end{array}$

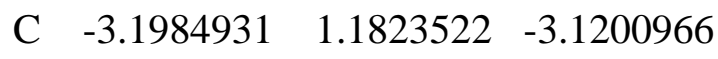
C $\quad-4.4552355 \quad 0.7314673 \quad-3.7117167$
C $\quad-4.4552355 \quad-0.7314673 \quad-3.7117167$
C $\quad-3.1786538 \quad 2.3164733 \quad-2.2946397$
$\begin{array}{llll}\text { C } & -2.3760014 & 2.3166309 & -1.0712386\end{array}$
$\begin{array}{llll}\text { C } & -3.1158858 & 3.0439256 & -0.0451308\end{array}$
$\begin{array}{llll}\text { C } & -4.3795352 & 3.4890131 & -0.6304535\end{array}$
$\begin{array}{lllll}\text { C } & -4.4140257 & 3.0434069 & -2.0231748\end{array}$
$\begin{array}{llll}\text { C } & -1.6297500 & 1.1819134 & -0.7248551\end{array}$
$\begin{array}{llll}\text { C } & -1.6543310 & 0.0000000 & -1.5828258\end{array}$
$\begin{array}{llll}\text { C } & -1.6297500 & -1.1819134 & -0.7248551\end{array}$
$\begin{array}{llll}\text { C } & -1.5891069 & -0.7306594 & 0.6632100\end{array}$
$\begin{array}{llll}\text { C } & -1.5891069 & 0.7306594 & 0.6632100\end{array}$
$\begin{array}{llll}\text { C } & -9.1474251 & 0.0000000 & 2.4989148\end{array}$
$\begin{array}{llll}\text { C } & -9.7839267 & 1.2239825 & 2.9183758\end{array}$
$\begin{array}{llll}\text { C } & -9.7839267 & -1.2239825 & 2.9183758\end{array}$
$\begin{array}{llll}\mathrm{N} & -10.2715445 & 2.2424527 & 3.2410909\end{array}$
$\begin{array}{llll}\mathrm{N} & -10.2715445 & -2.2424527 & 3.2410909\end{array}$

k. $8 \mathrm{C}_{\mathrm{s}}$

130

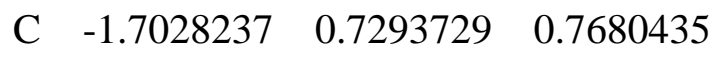

$\begin{array}{llll}\text { C } & -2.4866553 & 1.1970378 & 1.8929149\end{array}$ 

$\begin{array}{llll}\text { C } & -2.8901914 & 0.0000000 & 2.6952920\end{array}$
C $\quad-2.4866553-1.1970378 \quad 1.8929149$
$\begin{array}{llll}\text { C } & -1.7028237 & -0.7293729 & 0.7680435\end{array}$
$\begin{array}{llll}\text { C } & -6.5476329 & 0.7297890 & 2.6737604\end{array}$
$\begin{array}{llll}\text { C } & -6.5476329 & -0.7297890 & 2.6737604\end{array}$
$\begin{array}{llll}\text { C } & -5.2053425 & -1.1958863 & 2.9588087\end{array}$
$\begin{array}{llll}\text { C } & -4.3633103 & 0.0000000 & 3.2743108\end{array}$
$\begin{array}{llll}\text { C } & -5.2053425 & 1.1958863 & 2.9588087\end{array}$
C $\quad-5.5414868 \quad-3.0294650 \quad 1.3472865$
$\begin{array}{llll}\text { C } & -4.7077520 & -3.4776977 & 0.2350113\end{array}$
$\begin{array}{llll}\text { C } & -3.3401328 & -3.0296169 & 0.4824652\end{array}$
C $\quad-3.3176786 \quad-2.3042769 \quad 1.7465183$
$\begin{array}{llll}\text { C } & -4.6968868 & -2.3037606 & 2.2881647\end{array}$
$\begin{array}{llll}\text { C } & -3.0751515 & -2.6133177 & -1.9529909\end{array}$
$\begin{array}{llll}\text { C } & -2.5599835 & -1.4331076 & -2.6468248\end{array}$
C $\quad-1.7117819-0.6998614 \quad-1.7123503$
C $-1.7040992-1.4299951 \quad-0.4473561$
C $\quad-2.5399545-2.6089416 \quad-0.5937206$
$\begin{array}{llll}\text { C } & -2.5599835 & 1.4331076 & -2.6468248\end{array}$
$\begin{array}{llll}\text { C } & -3.0751515 & 2.6133177 & -1.9529909\end{array}$
C $\quad-2.5399545 \quad 2.6089416 \quad-0.5937206$
$\begin{array}{llll}\text { C } & -1.7040992 & 1.4299951 & -0.4473561\end{array}$
$\begin{array}{llll}\text { C } & -1.7117819 & 0.6998614 & -1.7123503\end{array}$
$\begin{array}{llll}\text { C } & -4.7077520 & 3.4776977 & 0.2350113\end{array}$
C $\quad-5.5414868 \quad 3.0294650 \quad 1.3472865$
$\begin{array}{llll}\text { C } & -4.6968868 & 2.3037606 & 2.2881647\end{array}$
$\begin{array}{llll}\text { C } & -3.3176786 & 2.3042769 & 1.7465183\end{array}$
$\begin{array}{llll}\text { C } & -3.3401328 & 3.0296169 & 0.4824652\end{array}$
$\begin{array}{llll}\text { C } & -7.3935732 & 2.6136429 & -0.2553212\end{array}$
$\begin{array}{llll}\text { C } & -8.2424517 & 1.4324779 & -0.4121941\end{array}$
$\begin{array}{llll}\text { C } & -8.2313978 & 0.7003410 & 0.8510195\end{array}$ 

C $\quad \begin{array}{llll}-7.3752234 & 1.4311295 & 1.7835628\end{array}$
$\begin{array}{llll}\text { C } & -6.8589914 & 2.6071891 & 1.1036692\end{array}$
$\begin{array}{llll}\text { C } & -8.2313978 & -0.7003410 & 0.8510195\end{array}$
$\begin{array}{llll}\text { C } & -8.2424517 & -1.4324779 & -0.4121941\end{array}$
C $\quad-7.3935732-2.6136429-0.2553212$
$\begin{array}{llll}\text { C } & -6.8589914 & -2.6071891 & 1.1036692\end{array}$
C $\quad-7.3752234 \quad-1.4311295 \quad 1.7835628$
C $\quad-6.5901979-3.0432637 \quad-1.3207059$
$\begin{array}{llll}\text { C } & -6.6028125 & -2.3163992 & -2.5860545\end{array}$
$\begin{array}{llll}\text { C } & -5.2408918 & -2.3160147 & -3.1211462\end{array}$
$\begin{array}{llll}\text { C } & -4.3882737 & -3.0433410 & -2.1865177\end{array}$
C $\quad-5.2204236-3.4879732-1.0687971$
$\begin{array}{llll}\text { C } & -4.7486287 & -1.1821512 & -3.7846170\end{array}$
$\begin{array}{llll}\text { C } & -5.5943985 & 0.0000000 & -3.9366909\end{array}$
$\begin{array}{llll}\text { C } & -4.7486287 & 1.1821512 & -3.7846170\end{array}$
$\begin{array}{llll}\text { C } & -3.3810108 & 0.7311385 & -3.5419050\end{array}$
$\begin{array}{llll}\text { C } & -3.3810108 & -0.7311385 & -3.5419050\end{array}$
$\begin{array}{llll}\text { C } & -5.2408918 & 2.3160147 & -3.1211462\end{array}$
$\begin{array}{llll}\text { C } & -6.6028125 & 2.3163992 & -2.5860545\end{array}$

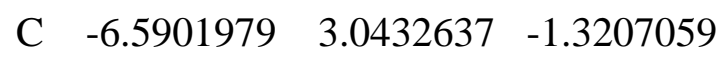
$\begin{array}{llll}\text { C } & -5.2204236 & 3.4879732 & -1.0687971\end{array}$
$\begin{array}{llll}\text { C } & -4.3882737 & 3.0433410 & -2.1865177\end{array}$
$\begin{array}{llll}\text { C } & -7.4151559 & 1.1823914 & -2.7364802\end{array}$
$\begin{array}{llll}\text { C } & -6.8989101 & 0.0000000 & -3.4225027\end{array}$
$\begin{array}{llll}\text { C } & -7.4151559 & -1.1823914 & -2.7364802\end{array}$
$\begin{array}{llll}\text { C } & -8.2508349 & -0.7308545 & -1.6265287\end{array}$
$\begin{array}{llll}\text { C } & -8.2508349 & 0.7308545 & -1.6265287\end{array}$
$\begin{array}{llll}\text { C } & -3.1346801 & 0.0000000 & 4.2389544\end{array}$
$\begin{array}{llll}\text { C } & -2.8602272 & 1.2249949 & 4.9482525\end{array}$
$\begin{array}{llll}\text { C } & -2.8602272 & -1.2249949 & 4.9482525\end{array}$
$\begin{array}{llll}\mathrm{N} & -2.6556367 & 2.2469658 & 5.4893515\end{array}$ 


$$
\begin{array}{lrrr}
\mathrm{N} & -2.6556367 & -2.2469658 & 5.4893515 \\
\mathrm{C} & 1.7028237 & 0.7293729 & 0.7680435 \\
\mathrm{C} & 2.4866553 & 1.1970378 & 1.8929149 \\
\mathrm{C} & 2.8901914 & 0.0000000 & 2.6952920 \\
\mathrm{C} & 2.4866553 & -1.1970378 & 1.8929149 \\
\mathrm{C} & 1.7028237 & -0.7293729 & 0.7680435 \\
\mathrm{C} & 6.5476329 & 0.7297890 & 2.6737604 \\
\mathrm{C} & 6.5476329 & -0.7297890 & 2.6737604 \\
\mathrm{C} & 5.2053425 & -1.1958863 & 2.9588087 \\
\mathrm{C} & 4.3633103 & 0.0000000 & 3.2743108 \\
\mathrm{C} & 5.2053425 & 1.1958863 & 2.9588087 \\
\mathrm{C} & 5.5414868 & -3.0294650 & 1.3472865 \\
\mathrm{C} & 4.7077520 & -3.4776977 & 0.2350113 \\
\mathrm{C} & 3.3401328 & -3.0296169 & 0.4824652 \\
\mathrm{C} & 3.3176786 & -2.3042769 & 1.7465183 \\
\mathrm{C} & 4.6968868 & -2.3037606 & 2.2881647 \\
\mathrm{C} & 3.0751515 & -2.6133177 & -1.9529909 \\
\mathrm{C} & 2.5599835 & -1.4331076 & -2.6468248 \\
\mathrm{C} & 1.7117819 & -0.6998614 & -1.7123503 \\
\mathrm{C} & 1.7040992 & -1.4299951 & -0.4473561 \\
\mathrm{C} & 2.5399545 & -2.6089416 & -0.5937206 \\
\mathrm{C} & 2.5599835 & 1.4331076 & -2.6468248 \\
\mathrm{C} & 3.0751515 & 2.6133177 & -1.9529909 \\
\mathrm{C} & 2.5399545 & 2.6089416 & -0.5937206 \\
\mathrm{C} & 1.7040992 & 1.4299951 & -0.4473561 \\
\mathrm{C} & 1.7117819 & 0.6998614 & -1.7123503 \\
\mathrm{C} & 4.7077520 & 3.4776977 & 0.2350113 \\
\mathrm{C} & 5.5414868 & 3.0294650 & 1.3472865 \\
\mathrm{C} & 4.6968868 & 2.3037606 & 2.2881647 \\
\mathrm{C} & 3.3176786 & 2.3042769 & 1.7465183 \\
\hline & 3.3401328 & 3.0296169 & 0.4824652
\end{array}
$$



C $\quad 7.3935732 \quad 2.6136429 \quad-0.2553212$
$\begin{array}{llll}\text { C } & 8.2424517 & 1.4324779 & -0.4121941\end{array}$
$\begin{array}{llll}\text { C } & 8.2313978 & 0.7003410 & 0.8510195\end{array}$
C $\quad 7.3752234 \quad 1.4311295 \quad 1.7835628$
$\begin{array}{llll}\text { C } & 6.8589914 & 2.6071891 & 1.1036692\end{array}$
$\begin{array}{llll}\text { C } & 8.2313978 & -0.7003410 & 0.8510195\end{array}$
$\begin{array}{llll}\text { C } & 8.2424517 & -1.4324779 & -0.4121941\end{array}$
$\begin{array}{llll}\text { C } & 7.3935732 & -2.6136429 & -0.2553212\end{array}$
$\begin{array}{llll}\text { C } & 6.8589914 & -2.6071891 & 1.1036692\end{array}$
C $7.3752234-1.4311295 \quad 1.7835628$
C $\quad 6.5901979-3.0432637 \quad-1.3207059$
$\begin{array}{llll}\text { C } & 6.6028125 & -2.3163992 & -2.5860545\end{array}$
$\begin{array}{llll}\text { C } & 5.2408918 & -2.3160147 & -3.1211462\end{array}$
$\begin{array}{llll}\text { C } & 4.3882737 & -3.0433410 & -2.1865177\end{array}$
$\begin{array}{llll}\text { C } & 5.2204236 & -3.4879732 & -1.0687971\end{array}$
C $\quad 4.7486287-1.1821512 \quad-3.7846170$
C $\quad 5.5943985 \quad 0.0000000 \quad-3.9366909$
$\begin{array}{llll}\text { C } & 4.7486287 & 1.1821512 & -3.7846170\end{array}$
C $\quad 3.3810108 \quad 0.7311385-3.5419050$
C $\quad 3.3810108-0.7311385-3.5419050$
$\begin{array}{llll}\text { C } & 5.2408918 & 2.3160147 & -3.1211462\end{array}$
$\begin{array}{llll}\text { C } & 6.6028125 & 2.3163992 & -2.5860545\end{array}$
C $\quad 6.5901979 \quad 3.0432637 \quad-1.3207059$
C $\quad 5.2204236 \quad 3.4879732 \quad-1.0687971$
$\begin{array}{llll}\text { C } & 4.3882737 & 3.0433410 & -2.1865177\end{array}$
C $\quad 7.4151559 \quad 1.1823914 \quad-2.7364802$
$\begin{array}{llll}\text { C } & 6.8989101 & 0.0000000 & -3.4225027\end{array}$
$\begin{array}{llll}\text { C } & 7.4151559 & -1.1823914 & -2.7364802\end{array}$
$\begin{array}{llll}\text { C } & 8.2508349 & -0.7308545 & -1.6265287\end{array}$
C $\quad 8.2508349 \quad 0.7308545 \quad-1.6265287$
$\begin{array}{llll}\text { C } & 3.1346801 & 0.0000000 & 4.2389544\end{array}$ 


$\begin{array}{lrrr}\mathrm{C} & 2.8602272 & 1.2249949 & 4.9482525 \\ \mathrm{C} & 2.8602272 & -1.2249949 & 4.9482525 \\ \mathrm{~N} & 2.6556367 & 2.2469658 & 5.4893515 \\ \mathrm{~N} & 2.6556367 & -2.2469658 & 5.4893515\end{array}$

\section{REFERENCES}

1. Iqbal, P.; Sun, S.; Hanwell, M. D.; Attwood, D.; Leggett, G. J.; Preece, J. A.; Richardson, T. H.; Tunnicliffe, D. Photochemical Fabrication of Three-Dimensional Micro- and Nano-Structured Surfaces from a C60 Monoadduct. J. Mater. Chem. 2008, 18, 2016-2021.

2. Xiao, Z.-Y.; Hou, J.-L.; Jiang, X.-K.; Li, Z.-T.; Ma, Z. Complexes between Hydrogen Bonded Bisporphyrin Tweezers and Cholesterol-Appended Fullerenes as Organogelators and Liquid Crystals. Tetrahedron 2009, 65, 10182-10191.

3. Modlińska, A.; Bauman, D. The Langmuir-Blodgett Technique as a Tool for Homeotropic Alignment of Fluorinated Liquid Crystals Mixed with Arachidic Acid. Int. J. Mol. Sci. 2011, 12, 4923.

4. Shubina, T. E.; Sharapa, D. I.; Schubert, C.; Zahn, D.; Halik, M.; Keller, P. A.; Pyne, S. G.; Jennepalli, S.; Guldi, D. M.; Clark, T. Fullerene Van Der Waals Oligomers as Electron Traps. J. Am. Chem. Soc. 2014, 136, 10890-10893.

5. Komatsu, K.; Wang, G.-W.; Murata, Y.; Tanaka, T.; Fujiwara, K.; Yamamoto, K.; Saunders, M. Mechanochemical Synthesis and Characterization of the Fullerene Dimer C120. J. Org. Chem. 1998, 63, 9358-9366.

6. Ohkubo, K.; Iwata, R.; Yanagimoto, T.; Fukuzumi, S. Enhanced Photoinduced Oligomerization of Fullerene Via Radical Coupling between Fullerene Radical Cation and Radical Anion Using 9-Mesityl10-Methylacridinium Ion. Chem. Commun. 2007, 3139-3141.

7. Maeyoshi, Y.; Saeki, A.; Suwa, S.; Omichi, M.; Marui, H.; Asano, A.; Tsukuda, S.; Sugimoto, M.; Kishimura, A.; Kataoka, K.; Seki, S. Fullerene Nanowires as a Versatile Platform for Organic Electronics. Sci. Rep. 2012, 2, 600.

8. Sheka, E. Fullerenes: Nanochemistry, Nanomagnetism, Nanomedicine, Nanophotonics. CRC Press (an imprint of Taylor \& Francis Group, Boca Raton, Florida, United States of America). 2011, ISBN 978-1-4398-0642-5. 
